\title{
Status of the Higgs singlet extension of the standard model after $L H C$ run 1
}

\author{
Tania Robens ${ }^{1, a}$, Tim Stefaniak ${ }^{2, b}$ \\ ${ }^{1}$ Institut für Kern- und Teilchenphysik, TU Dresden, Zellescher Weg 19, 01069 Dresden, Germany \\ 2 Department of Physics, Santa Cruz Institute for Particle Physics, University of California, Santa Cruz, CA 95064, USA
}

Received: 19 January 2015 / Accepted: 17 February 2015 / Published online: 4 March 2015

(C) The Author(s) 2015. This article is published with open access at Springerlink.com

\begin{abstract}
We discuss the current status of theoretical and experimental constraints on the real Higgs singlet extension of the standard model. For the second neutral (non-standard) Higgs boson we consider the full mass range from $1 \mathrm{GeV}$ to $1 \mathrm{TeV}$ accessible at past and current collider experiments. We separately discuss three scenarios, namely, the case where the second Higgs boson is lighter than, approximately equal to, or heavier than the discovered Higgs state at around $125 \mathrm{GeV}$. We investigate the impact of constraints from perturbative unitarity, electroweak precision data with a special focus on higher-order contributions to the $W$ boson mass, perturbativity of the couplings as well as vacuum stability. The latter two are tested up to a scale of $\sim 4 \times 10^{10} \mathrm{GeV}$ using renormalization group equations. Direct collider constraints from Higgs signal rate measurements at the LHC and $95 \%$ confidence level exclusion limits from Higgs searches at LEP, Tevatron, and LHC are included via the public codes HiggsSignals and HiggsBounds, respectively. We identify the strongest constraints in the different regions of parameter space. We comment on the collider phenomenology of the remaining viable parameter space and the prospects for a future discovery or exclusion at the LHC.
\end{abstract}

\section{Contents}

1 Introduction .................. 1

2 The model ............... . . 2

2.1 Potential and couplings .......... . . . . 2

2.2 Model parameters . . . . . . . . . . . . 4

3 Theoretical and experimental constraints . . . . . 4

3.1 Perturbative unitarity .......... . . . 4

3.2 Perturbativity of the couplings ...... 5

a e-mail: Tania.Robens@tu-dresden.de

be-mail: tistefan@ucsc.edu
3.3 Renormalization group equation evolution of the couplings . . . . . . . . . . 5

3.4 The $W$ boson mass and electroweak oblique parameters $S, T, U \ldots \ldots$

3.5 Exclusion limits from Higgs searches at LEP and LHC . . . . . . . . . . . . . . . . . 7

3.6 Higgs boson signal rates measured at the LHC . 8

4 Results of the full parameter scan ... . . . . . . 11

4.1 High mass region . . . . . . . . . . . . . 11

4.2 Low mass region . . . . . . . . . . . . . . 17

4.3 Intermediate mass region . . . . . . . . . 20

5 Conclusions ................ . 21

Appendix A: Minimization and vacuum stability

conditions ................ 23

Appendix B: RGEs for SM gauge couplings and the top

quark Yukawa coupling ............. 23

References ................. 24

\section{Introduction}

The LHC discovery [1,2] of a Higgs boson in July 2012 has been a major breakthrough in modern particle physics. The first runs of the LHC at 7 and $8 \mathrm{TeV}$ are now completed and the main results from various experimental analyses of the Higgs boson properties have been presented at the 2014 summer conferences. So far, the discovered state is well compatible [3-10] with the interpretation in terms of the scalar boson of the standard model (SM) Higgs mechanism [1115]. A simple combination of the Higgs mass measurements performed by ATLAS [16] and CMS [17] yields a central value of

$m_{H}=(125.14 \pm 0.24) \mathrm{GeV}$

If the discovered particle is indeed the Higgs boson predicted by the SM, its mass constitutes the last unknown 
ingredient to this model, as all other properties of the electroweak sector then follow directly from theory. The current and future challenge for the theoretical and experimental community is to thoroughly investigate the Higgs boson's properties in order to identify whether the SM Higgs sector is indeed complete, or instead, the structure of a more involved Higgs sector is realized. On the experimental side, this requires detailed and accurate measurements of its coupling strengths and $\mathcal{C P}$ structure at the LHC and ultimately at future experimental facilities for Higgs boson precision studies, such as the International Linear Collider (ILC) [18]. A complementary and equally important strategy is to perform collider searches for additional Higgs bosons. Such a finding would provide clear evidence for a non-minimal Higgs sector. This road needs to be continued within the full mass range that is accessible to current and future experiments.

In this work, we consider the simplest extension of the SM Higgs sector, where an additional real singlet field is added, which is neutral under all quantum numbers of the SM gauge group $[19,20]$ and acquires a vacuum expectation value (VEV). This model has been widely studied in the literature [21-48]. Here, we present a complete exploration of the model parameter space in the light of the latest experimental and theoretical constraints. We consider masses of the second (non-standard) Higgs boson in the whole mass range up to $1 \mathrm{TeV}$, thus extending and updating the findings of previous work [41]. This minimal setup can be interpreted as a limiting case for more generic BSM scenarios, e.g. models with additional gauge sectors [49] or additional matter content [50,51].

In our analysis, we study the implications of various constraints: We take into account bounds from perturbative unitarity and electroweak (EW) precision measurements, in particular focussing on higher-order corrections to the $W$ boson mass [43]. Furthermore, we study the impact of requiring perturbativity, vacuum stability, and a correct minimization of the model up to a high energy scale using renormalization group evolved couplings. ${ }^{1}$ We include the exclusion limits from Higgs searches at the LEP, Tevatron and LHC experiments via the public tool HiggsBounds [52-55], and use the program HiggsSignals [56] (cf. also Ref. [57]) to test the compatibility of the model with the signal strength measurements of the discovered Higgs state.

We separate the discussion of the parameter space into three different mass regions:

(i) the high mass region, $m_{H} \in[130,1000] \mathrm{GeV}$, where the lighter Higgs boson $h$ is interpreted as the discovered Higgs state;

\footnotetext{
1 The value of this high energy scale is chosen to be larger than the energy scale where the running SM Higgs quartic coupling turns negative. This will be made more precise in Sect. 3 .
}

(ii) the intermediate mass region, where both Higgs bosons $h$ and $H$ are located in the mass region $[120,130] \mathrm{GeV}$ and potentially contribute to the measured signal rates, and

(iii) the low mass region, $m_{h} \in[1,120] \mathrm{GeV}$, where the heavier Higgs boson $H$ is interpreted as the discovered Higgs state.

We find that the most severe constraints in the whole parameter space for the second Higgs mass $m_{H} \lesssim 300 \mathrm{GeV}$ are mostly given by limits from collider searches for a SM Higgs boson as well as by the LHC Higgs boson signal strength measurements. For $m_{H} \gtrsim 300 \mathrm{GeV}$ limits from higher-order contributions to the $W$ boson mass prevail, followed by the requirement of perturbativity of the couplings which is tested via renormalization group equation (RGE) evolution. For the remaining viable parameter space we present predictions for signal cross sections of the yet undiscovered second Higgs boson for the LHC at center-of-mass (CM) energies of 8 and $14 \mathrm{TeV}$, discussing both the SM Higgs decay signatures and the novel Higgs-to-Higgs decay mode $H \rightarrow h h$. We furthermore present our results in terms of a global suppression factor $\kappa$ for SM-like channels as well as the total width $\Gamma$ of the second Higgs boson, and show regions which are allowed in the $(\kappa, \Gamma)$ plane.

The paper is organized as follows: in Sect. 2 we briefly review the model and the chosen parametrization. In Sect. 3 we elaborate upon the various theoretical and experimental constraints and discuss their impact on the model parameter space. In Sect. 4 a scan of the full model parameter space is presented, in which all relevant constraints are combined. This is followed by a discussion of the collider phenomenology of the viable parameter space. We summarize and conclude in Sect. 5.

\section{The model}

\subsection{Potential and couplings}

The real Higgs singlet extension of the SM is described in detail in Refs. [19,20,41,58]. Here, we only briefly review the theoretical setup as well as the main features relevant to the work presented here.

We consider the extension of the SM electroweak sector containing a complex $S U(2)_{L}$ doublet, in the following denoted by $\Phi$, by an additional real scalar $S$ which is a singlet under all SM gauge groups. The most generic renormalizable Lagrangian is then given by

$\mathscr{L}_{s}=\left(D^{\mu} \Phi\right)^{\dagger} D_{\mu} \Phi+\partial^{\mu} S \partial_{\mu} S-V(\Phi, S)$,

with the scalar potential 


$$
\begin{aligned}
V(\Phi, S)= & -m^{2} \Phi^{\dagger} \Phi-\mu^{2} S^{2} \\
& +\left(\Phi^{\dagger} \Phi S^{2}\right)\left(\begin{array}{cc}
\lambda_{1} & \frac{\lambda_{3}}{2} \\
\frac{\lambda_{3}}{2} \lambda_{2}
\end{array}\right)\left(\begin{array}{c}
\Phi^{\dagger} \Phi \\
S^{2}
\end{array}\right) \\
= & -m^{2} \Phi^{\dagger} \Phi-\mu^{2} S^{2}+\lambda_{1}\left(\Phi^{\dagger} \Phi\right)^{2}+\lambda_{2} S^{4} \\
& +\lambda_{3} \Phi^{\dagger} \Phi S^{2} .
\end{aligned}
$$

Here, we implicitly impose a $Z_{2}$ symmetry which forbids all linear or cubic terms of the singlet field $S$ in the potential. The scalar potential $V(\Phi, S)$ is bounded from below if the following conditions are fulfilled:

$4 \lambda_{1} \lambda_{2}-\lambda_{3}^{2}>0$

$\lambda_{1}, \lambda_{2}>0$,

cf. Appendix A. If the first condition, Eq. (4), is fulfilled, the extremum is a local minimum. The second condition, Eq. (5), guarantees that the potential is bounded from below for large field values. We assume that both Higgs fields $\Phi$ and $S$ have a non-zero VEV, denoted by $v$ and $x$, respectively. In the unitary gauge, the Higgs fields are given by

$\Phi \equiv\left(\begin{array}{l}0 \\ \frac{\tilde{h}+v}{\sqrt{2}}\end{array}\right), \quad S \equiv \frac{h^{\prime}+x}{\sqrt{2}}$.

Expansion around the minimum leads to the squared mass matrix

$\mathcal{M}^{2}=\left(\begin{array}{cc}2 \lambda_{1} v^{2} & \lambda_{3} v x \\ \lambda_{3} v x & 2 \lambda_{2} x^{2}\end{array}\right)$

with the mass eigenvalues

$$
\begin{gathered}
m_{h}^{2}=\lambda_{1} v^{2}+\lambda_{2} x^{2}-\sqrt{\left(\lambda_{1} v^{2}-\lambda_{2} x^{2}\right)^{2}+\left(\lambda_{3} x v\right)^{2}}, \\
m_{H}^{2}=\lambda_{1} v^{2}+\lambda_{2} x^{2}+\sqrt{\left(\lambda_{1} v^{2}-\lambda_{2} x^{2}\right)^{2}+\left(\lambda_{3} x v\right)^{2}},
\end{gathered}
$$

where $h$ and $H$ are the scalar fields with masses $m_{h}$ and $m_{H}$ respectively, and $m_{h}^{2} \leq m_{H}^{2}$ by convention. Note that $m_{h}^{2} \geq 0$ follows from Eq. (4) and we assume Eqs. (4) and (5) to be fulfilled in all following definitions. The gauge and mass eigenstates are related via the mixing matrix

$$
\left(\begin{array}{c}
h \\
H
\end{array}\right)=\left(\begin{array}{cc}
\cos \alpha & -\sin \alpha \\
\sin \alpha & \cos \alpha
\end{array}\right)\left(\begin{array}{c}
\tilde{h} \\
h^{\prime}
\end{array}\right),
$$

where the mixing angle $-\frac{\pi}{2} \leq \alpha \leq \frac{\pi}{2}$ is given by

$$
\begin{aligned}
\sin 2 \alpha & =\frac{\lambda_{3} x v}{\sqrt{\left(\lambda_{1} v^{2}-\lambda_{2} x^{2}\right)^{2}+\left(\lambda_{3} x v\right)^{2}}}, \\
\cos 2 \alpha & =\frac{\lambda_{2} x^{2}-\lambda_{1} v^{2}}{\sqrt{\left(\lambda_{1} v^{2}-\lambda_{2} x^{2}\right)^{2}+\left(\lambda_{3} x v\right)^{2}}} .
\end{aligned}
$$

It follows from Eq. (10) that the light (heavy) Higgs boson couplings to SM particles are suppressed by $\cos \alpha(\sin \alpha)$.
Using Eqs. (8), (9), and (11), we can express the couplings in terms of the mixing angle $\alpha$, the Higgs VEVs $x$ and $v$ and the two Higgs boson masses, $m_{h}$ and $m_{H}$ :

$\lambda_{1}=\frac{m_{h}^{2}}{2 v^{2}}+\frac{\left(m_{H}^{2}-m_{h}^{2}\right)}{2 v^{2}} \sin ^{2} \alpha=\frac{m_{h}^{2}}{2 v^{2}} \cos ^{2} \alpha+\frac{m_{H}^{2}}{2 v^{2}} \sin ^{2} \alpha$,

$\lambda_{2}=\frac{m_{h}^{2}}{2 x^{2}}+\frac{\left(m_{H}^{2}-m_{h}^{2}\right)}{2 x^{2}} \cos ^{2} \alpha=\frac{m_{h}^{2}}{2 x^{2}} \sin ^{2} \alpha+\frac{m_{H}^{2}}{2 x^{2}} \cos ^{2} \alpha$,

$\lambda_{3}=\frac{\left(m_{H}^{2}-m_{h}^{2}\right)}{2 v x} \sin (2 \alpha)$.

If kinematically allowed, the additional decay channel $H \rightarrow h h$ is present. Its partial decay width is given by $[19,58]$

$\Gamma_{H \rightarrow h h}=\frac{\left|\mu^{\prime}\right|^{2}}{8 \pi m_{H}} \sqrt{1-\frac{4 m_{h}^{2}}{m_{H}^{2}}}$,

where the coupling strength $\mu^{\prime}$ of the $H \rightarrow h h$ decay reads

$\mu^{\prime}=-\frac{\sin (2 \alpha)}{2 v x}(\sin \alpha v+\cos \alpha x)\left(m_{h}^{2}+\frac{m_{H}^{2}}{2}\right)$.

We therefore obtain as branching ratios for the heavy Higgs mass eigenstate $m_{H}$

$$
\begin{aligned}
\mathrm{BR}_{H \rightarrow h h} & =\frac{\Gamma_{H \rightarrow h h}}{\Gamma_{\text {tot }}}, \\
\mathrm{BR}_{H \rightarrow \mathrm{SM}} & =\sin ^{2} \alpha \times \frac{\Gamma_{\mathrm{SM}, H \rightarrow \mathrm{SM}}}{\Gamma_{\text {tot }}},
\end{aligned}
$$

where $\Gamma_{\mathrm{SM}} H \rightarrow \mathrm{SM}$ denotes the partial decay width of the SM Higgs boson and $H \rightarrow$ SM represents any SM Higgs decay mode. The total width is given by

$\Gamma_{\text {tot }}=\sin ^{2} \alpha \times \Gamma_{\mathrm{SM}}$ tot $+\Gamma_{H \rightarrow h h}$,

where $\Gamma_{\mathrm{SM}}$, tot denotes the total width of the SM Higgs boson with mass $m_{H}$. The suppression by $\sin ^{2} \alpha$ directly follows from the suppression of all SM-like couplings, cf. Eq. (10). For $\mu^{\prime}=0$, we recover the SM Higgs boson branching ratios.

For collider phenomenology, two features are important:

- the suppression of the production cross section of the two Higgs states induced by the mixing, which is given by $\sin ^{2} \alpha\left(\cos ^{2} \alpha\right)$ for the heavy (light) Higgs, respectively;

- the suppression of the Higgs decay modes to SM particles, which is realized if the competing decay mode $H \rightarrow h h$ is kinematically accessible.

For the high mass (low mass) scenario, i.e. the case where the light (heavy) Higgs boson is identified with the discovered Higgs state at $\sim 125 \mathrm{GeV},|\sin \alpha|=0$ (1) corresponds to the complete decoupling of the second Higgs boson and therefore the SM-like scenario. 


\subsection{Model parameters}

At the Lagrangian level, the model has five free parameters,

$\lambda_{1}, \lambda_{2}, \lambda_{3}, v, x$

while the values of the additional parameters $\mu^{2}, m^{2}$ are fixed by the minimization conditions to the values

$$
\begin{aligned}
& m^{2}=\lambda_{1} v^{2}+\frac{\lambda_{3}}{2} x^{2}, \\
& \mu^{2}=\lambda_{2} x^{2}+\frac{\lambda_{3}}{2} v^{2},
\end{aligned}
$$

cf. Appendix A. In this work, we choose to parametrize the model in terms of the independent physical quantities

$m_{h}, m_{H}, \alpha, v, \tan \beta \equiv \frac{v}{x}$.

The couplings $\lambda_{1}, \lambda_{2}$, and $\lambda_{3}$ can then be expressed via Eq. (13). The VEV of the Higgs doublet $\Phi$ is given by the SM value $v \sim 246 \mathrm{GeV}$. Unless otherwise stated, we fix one of the Higgs masses to be $m_{h / H}=125.14 \mathrm{GeV}$, hence interpreting the Higgs boson $h / H$ as the discovered Higgs state at the LHC. In this case, we are left with only three independent parameters, $m \equiv m_{H / h}$, $\sin \alpha, \tan \beta$, where the latter enters the collider phenomenology only via the additional decay channel $^{2} H \rightarrow h h$.

\section{Theoretical and experimental constraints}

We now discuss the various theoretical and experimental constraints on the singlet extension model. In our analysis, we impose the following constraints:

(1) limits from perturbative unitarity,

(2) limits from $\mathrm{EW}$ precision data in form of the $S, T, U$ parameters [59-62] as well as the singlet-induced NLO corrections to the $W$ boson mass as presented in Ref. [43],

(3) perturbativity of the couplings as well as the requirement on the potential to be bounded from below, Eqs. (4) and (5),

(4) limits from perturbativity of the couplings as well as vacuum stability up to a certain scale $\mu_{\text {run }}$, where we chose $\mu_{\text {run }} \sim 4 \times 10^{10} \mathrm{GeV}$ as benchmark point (these constraints will only be applied in the high mass region; see Sect. 3.3 for further discussion),

\footnotetext{
${ }^{2}$ In fact, all Higgs self-couplings depend on $\tan \beta$. However, in the factorized leading-order description of production and decay followed here, and as long as no experimental data exists which constrains the Higgs boson self-couplings, only the $H h h$ coupling needs to be considered.
}

(5) upper cross section limits at 95\% confidence level (CL) from null results in Higgs searches at the LEP, Tevatron and LHC experiments,

(6) consistency with the Higgs boson signal rates measured at the LHC experiments.

The constraints (1)-(4) have already been discussed extensively in a previous publication [41], where the scan was, however, restricted to the case that $m_{H} \geq 600 \mathrm{GeV}$. In the following, we will therefore briefly recall the definition of the theoretically motivated bounds and comment on their importance in the whole mass range $m_{h / H} \in[1,1000] \mathrm{GeV}$.

\subsection{Perturbative unitarity}

Tree-level perturbative unitarity $[63,64]$ puts a constraint on the Higgs masses via a relation on the partial wave amplitudes $a_{J}(s)$ of all possible $2 \rightarrow 2$ scattering processes:

$\left|\operatorname{Re}\left(a_{J}(s)\right)\right| \leq \frac{1}{2}$

where the partial wave amplitude $a_{0}$ poses the strongest constraint. Following Ref. [41], we consider all $2 \rightarrow 2$ processes $X_{1} X_{2} \rightarrow Y_{1} Y_{2}$, with $\left(X_{1}, X_{2}\right),\left(Y_{1}, Y_{2}\right) \in$ $\left(W^{+} W^{-}, Z Z, h h, h H, H H\right)$, and impose the condition of Eq. (21) to the eigenvalues of the diagonalized scattering matrix. Note that the unitarity constraint based on the consideration of $W_{L} W_{L} \rightarrow W_{L} W_{L}$ scattering alone, leading to $m_{H} \lesssim 700 \mathrm{GeV}$ (as e.g. in Ref. [26]), is much loosened when all scattering channels are taken into account $[58,65]$.

In general, perturbative unitarity poses an upper limit on $\tan \beta$. In the decoupling case, which corresponds to $\sin \alpha \rightarrow$ 0 (1) for the light (heavy) Higgs being SM-like, it is given by [41]

$$
\tan ^{2} \beta \leq \frac{16 \pi v^{2}}{3 m^{2}}+\mathcal{O}(\alpha) \text { for } a_{0}(h h \rightarrow h h) \leq 0.5
$$

where $h$ and $m$ refer to the purely singlet Higgs state and its respective mass.

While in the high mass scenario this bound is always superseded by bounds from perturbativity of the couplings, cf. Sect. 3.3, in the low mass scenario this poses the strongest theoretical bound on $\tan \beta$. We exemplarily show the upper limits on $\tan \beta$ derived from perturbative unitarity in Fig. 1 for the low mass range $m \in[20,120]$ for the four values of $\sin \alpha=1.0,0.9,0.5,0.0$. The bounds on $\tan \beta$ are strongest for small values of $\sin \alpha$. However, values too far from the decoupling case $\sin \alpha \approx 1$ are highly constrained by Higgs searches at LEP as well as by the LHC signal strength measurements of the heavier Higgs at $\sim 125 \mathrm{GeV}$, cf. Sects. 3.5 and 3.6 for more details. 


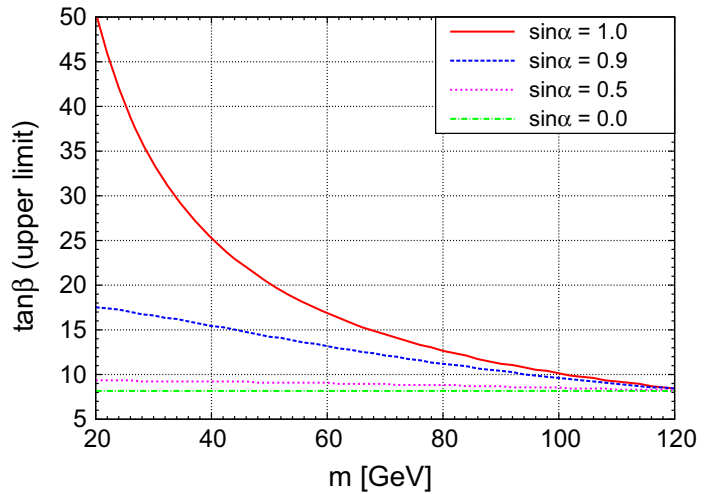

Fig. 1 Maximally allowed values for $\tan \beta$ in the low mass range, $m \in$ $[20,120] \mathrm{GeV}$, for various values of $\sin \alpha=1.0,0.9,0.5,0.0$, from considering only perturbative unitarity

\subsection{Perturbativity of the couplings}

For perturbativity of the couplings, we require that

$\left|\lambda_{i}\right| \leq 4 \pi, \quad i \in(1,2,3)$.

At the electroweak scale, these bounds do not pose additional constraints on the parameter space after the limits from perturbative unitarity have been taken into account.

\subsection{Renormalization group equation evolution of the couplings}

While perturbativity as well as vacuum stability and the existence of a local minimum at the electroweak scale are necessary ingredients for the validity of a parameter point, it is instructive to investigate up to which energy scale these requirements remain valid. In particular, we study whether the potential is bounded from below and features a local minimum at energy scales above the electroweak scale. In order to achieve this, we promote the requirements of Eqs. (4), (5) and (23) to be valid at an arbitrary scale $\mu_{\text {run }}$, where $\lambda_{i}\left(\mu_{\text {run }}\right)$ are evolved according to the one-loop RGEs (see e.g. Ref. [66])

$$
\begin{aligned}
\frac{d}{d t} \lambda_{1}= & \frac{1}{16 \pi^{2}}\left\{\frac{1}{4} \lambda_{3}^{2}+12 \lambda_{1}^{2}+6 \lambda_{1} y_{t}^{2}-3 y_{t}^{4}\right. \\
& \left.-\frac{3}{2} \lambda_{1}\left(3 g^{2}+g_{1}^{2}\right)+\frac{3}{16}\left[2 g^{4}+\left(g^{2}+g_{1}^{2}\right)^{2}\right]\right\},
\end{aligned}
$$

$$
\begin{aligned}
\frac{d}{d t} \lambda_{2}= & \frac{1}{16 \pi^{2}}\left[\lambda_{3}^{2}+9 \lambda_{2}^{2}\right] \\
\frac{d}{d t} \lambda_{3}= & \frac{1}{16 \pi^{2}} \lambda_{3}\left[6 \lambda_{1}+3 \lambda_{2}+2 \lambda_{3}+3 y_{t}^{2}\right. \\
& \left.-\frac{3}{4}\left(3 g^{2}+g_{1}^{2}\right)\right] .
\end{aligned}
$$

Here we introduced $t=2 \log \left(\mu_{\mathrm{run}} / v\right)$ as a dimensionless running parameter. The initial conditions at the electroweak scale require that $\lambda_{i}\left(\mu_{\text {res }} \equiv v\right)$ are given by Eq. (13). The top Yukawa coupling $y_{t}$ as well as the SM gauge couplings $g, g_{1}$ evolve according to the one-loop SM RGEs, cf. Appendix B. For the decoupling case as well as to cross-check the implementation of the running of the gauge couplings we reproduced the results of Ref. [67].

As in Ref. [41], we require all RGE-dependent constraints to be valid at a scale which is slightly higher than the breakdown scale of the SM, such that the singlet extension of the SM improves the stability of the electroweak vacuum. The SM breakdown scale is defined as the scale where the potential becomes unbounded from below in the decoupled, SMlike scenario. With the input values of $m_{h}=125.14 \mathrm{GeV}$ and $v=246.22 \mathrm{GeV}$, a top mass of $173.0 \mathrm{GeV}$ as well as a top-Yukawa coupling $y_{t}\left(m_{t}\right)=0.93587$ and strong coupling constant $\alpha_{s}\left(m_{Z}\right)=0.1184$, we obtain as a SM breakdown scale $^{3}$

$\mu_{\text {run, SM bkdw }} \sim 2.5 \times 10^{10} \mathrm{GeV}$.

We therefore chose as a slightly higher test scale the value $\mu_{\text {run, stab }} \sim 4.0 \times 10^{10} \mathrm{GeV}$. Naturally, we only apply this test to points in the parameter space which have passed constraints from perturbative unitarity as well as perturbativity of the couplings at the electroweak scale. Changing the scale to higher (lower) values leads to more (less) constrained regions in the models parameter space [41].

In the high mass scenario we see the behavior studied in Ref. [41] for Higgs masses $\geq 600 \mathrm{GeV}$ continuing to the lower mass ranges. The strongest constraints that impact different parts of the $(\sin \alpha, \tan \beta)$ parameter space are displayed in Fig. 2 for a heavy Higgs mass of $m_{H}=600 \mathrm{GeV}$ (taken from Ref. [41]). Two main features can be observed:

First, the upper value of $\tan \beta$ for fixed Higgs masses is determined by requiring perturbativity of $\lambda_{2}$ as well as perturbative unitarity, cf. Sect. 3.1.

Second, the allowed range of the mixing angle $\sin \alpha$ is determined by perturbativity of the couplings as well as the requirement of vacuum stability, especially when these are required at renormalization scales $\mu_{\text {run }}$, which are significantly larger than the electroweak scale. Small mixings are

\footnotetext{
${ }^{3}$ As has been discussed in e.g. Ref. [67], the scale where $\lambda_{1}=0$ in the decoupling case strongly depends on the initial input parameters. However, as we are only interested in the difference of the running in the case of a non-decoupled singlet component with respect to the standard model, we do not need to determine this scale to the utmost precision. For a more thorough discussion of the behavior of the RGE-resulting constraints in case of varying input parameters, see e.g. Ref. [41].
} 


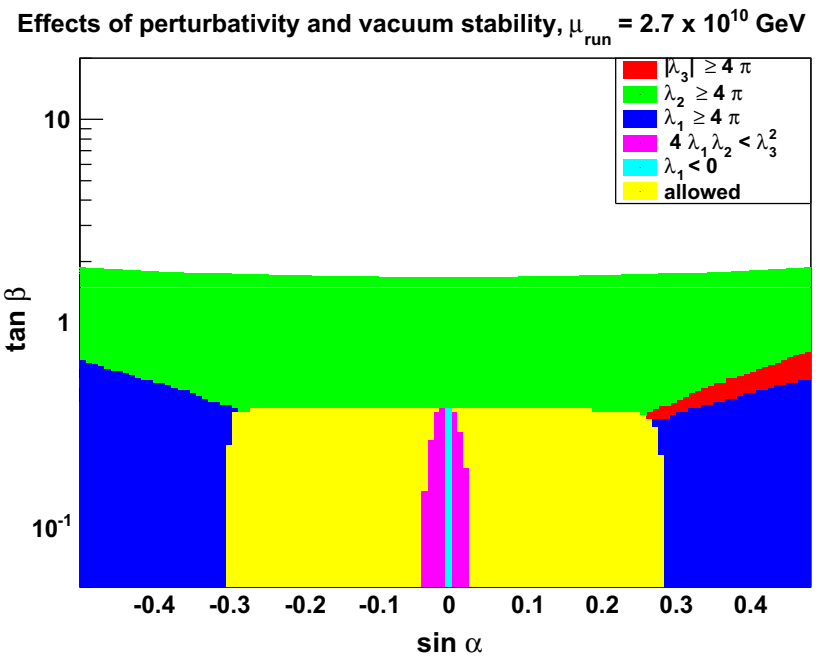

Fig. 2 Limits in the $(\sin \alpha, \tan \beta)$ plane for $m_{H}=600 \mathrm{GeV}$ from requiring perturbativity and vacuum stability at a scale $\mu_{\text {run }}=2.7 \times$ $10^{10} \mathrm{GeV}$ using RGE evolution. Taken from Ref. [41]

excluded by the requirements of vacuum stability ${ }^{4}$ as well as minimization of the scalar potential. This corresponds to the fact that we enter an unstable vacuum for $\mu_{\text {run }} \gtrsim \mu_{\text {SM,bkdw }}$ for $\sin \alpha \sim 0$.

In summary, the constraints from RGE evolution of the couplings pose the strongest bounds on the minimally allowed value $|\sin \alpha|$ and the maximal value of $\tan \beta$ in the high mass scenario. Note that, for lower $m_{H}$, the $(\sin \alpha, \tan \beta$ ) parameter space is less constrained, as will be discussed in Sect. 4.1.

In the low mass scenario, i.e. where the heavier Higgs state is considered to be the discovered Higgs boson, none of the points in our scan fulfilled vacuum stability above the electroweak scale. This is due to the fact that for a relatively low $m_{h}$, the value of $\lambda_{1}$ at the electroweak scale is quite small, cf. Eq. (13). In the non-decoupled case, $|\sin \alpha| \neq 1, \lambda_{1}$ then receives negative contributions in the RG evolution toward higher scales, leading to $\lambda_{1}\left(\mu_{\text {run }}\right) \leq 0$ already at relatively low scales $\mu_{\text {run }}$, corresponding to the breakdown of the electroweak vacuum. Hence, in the low mass scenario, the theory breaks down even earlier than in the SM case. In the analysis presented here, we will therefore refrain from taking the limits from RGE running into account in the low mass scenario. Then the theoretically maximally allowed value of $\tan \beta$ is

\footnotetext{
${ }^{4}$ For the requirement of vacuum stability, we found that in some cases the coupling strengths vary very mildly over large variations of the RGE running scale. In these regions the inclusion of higher-order corrections in the spirit of Ref. [67] seems indispensable. Therefore, all lower limits on the mixing angle originating from RGE constraints need to be viewed in this perspective. In fact, such higher-order contributions to the scalarextended RGEs have recently been presented in Ref. [68]. However, the authors did not specifically investigate the higher-order effects on parameter points which exhibit small variations over large energy scales at NLO.
}

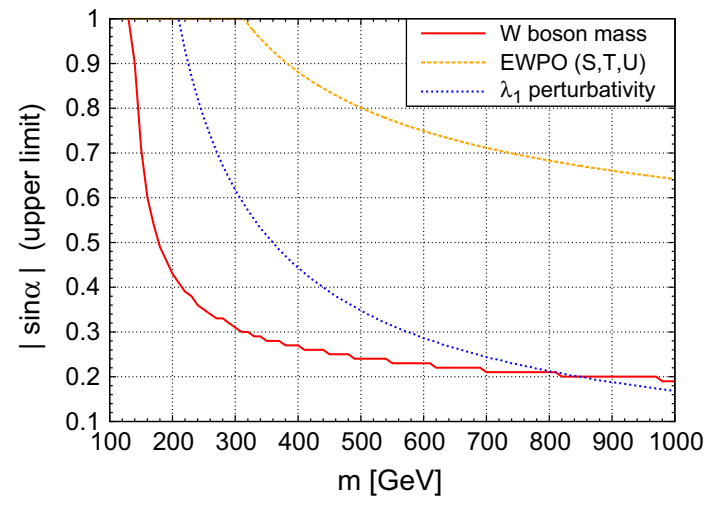

Fig. 3 Maximal allowed values for $|\sin \alpha|$ in the high mass region, $m_{H} \in[130,1000] \mathrm{GeV}$, from NLO calculations of the $W$ boson mass (red, solid) [43], electroweak precision observables (EWPOs) tested via the oblique parameters $S, T$, and $U$ (orange, dashed), as well as from the perturbativity requirement of the RG-evolved coupling $\lambda_{1}$ (blue, dotted), evaluated at $\tan \beta=0.1$. For Higgs masses $m_{H} \lesssim 800 \mathrm{GeV}$ the NLO corrections to the $W$ boson mass yield the strongest constraint

determined from perturbative unitarity and rises to quite large values, where we obtain $\tan \beta_{\max } \lesssim 50$, depending on the value of the light Higgs mass $m_{h}$.

Further constraints on $\tan \beta$ in the low mass scenario stem from the Higgs signal rate observables through the potential decay $H \rightarrow h h$, as will be discussed in Sect. 3.6.

\subsection{The $W$ boson mass and electroweak oblique parameters $S, T, U$}

Recently, the one-loop corrections to the $W$ boson mass, $m_{W}$, for this model have been calculated in Ref. [43]. In that analysis, $m_{W}$ is required to agree within $2 \sigma$ with the experimental value $m_{W}^{\exp }=80.385 \pm 0.015 \mathrm{GeV}$ [69-71], leading to an allowed range for the purely singlet-induced corrections of $\Delta m_{W}^{\text {sing }} \in[-5 \mathrm{MeV} ; 55 \mathrm{MeV}]$. Theoretical uncertainties due to contributions at even higher orders have been estimated to be $\mathcal{O}(1 \mathrm{MeV})$. The one-loop corrections are independent ${ }^{5}$ of $\tan \beta$ and give rise to additional constraints on $|\sin \alpha|$, which in the high mass scenario turn out to be much more stringent [43] than the constraints obtained from the oblique parameters $S, T$, and $U$ [59-62].

Figure 3 shows the maximally allowed mixing angle obtained from the $m_{W}$ constraint as a function of the heavy Higgs mass $m_{H}$ in the high mass scenario. For comparison, we also included the limit stemming from the electroweak oblique parameters $S, T$, and $U$ (see below), as well as from requiring perturbativity of $\lambda_{1}\left(\mu_{\text {run }}\right)$, evaluated at $\tan \beta=0.1$. We see that for $m_{H} \lesssim 800 \mathrm{GeV}$ constraints from $m_{W}$ yield the strongest constraint. The oblique param-

\footnotetext{
5 In the electroweak gauge sector, $\tan \beta$ only enters at the 2-loop level when the Higgs mass sector is renormalized in the on-shell scheme.
} 
eters $S, T$, and $U$ do not pose additional limits on the allowed parameter space.

In the low mass region, as discussed in Ref. [43], the NLO contributions within the Higgs singlet extension model even tend to decrease the current $\sim 20 \mathrm{MeV}$ discrepancy between the theoretical value $m_{W}$ in the SM [72] and the experimental measurement [69-71]. However, substantial reduction of the discrepancy only occurs if the light Higgs has a sizable doublet component. Hence, this possibility is strongly constrained by exclusion limits from LEP and/or LHC Higgs searches (depending on the light Higgs mass) as well as by the LHC Higgs signal rate measurements.

In the low mass region the electroweak oblique parameters pose non-negligible constraints, as will be shown in Sect. 4.2. However, these constraints are again superseded once the Higgs signal strength as well as direct search limits from LEP are taken into account, cf. Sects. 3.5 and 3.6 respectively.

In our analysis we test the constraints from the electroweak oblique parameters $S, T$, and $U$ by evaluating

$\chi_{\mathrm{STU}}^{2}=\mathbf{x}^{T} \mathbf{C}^{-1} \mathbf{x}$

with $\mathbf{x}^{T}=(S-\hat{S}, T-\hat{T}, U-\hat{U})$, where the observed parameters are given by [73]

$\hat{S}=0.05, \quad \hat{T}=0.09, \quad \hat{U}=0.01$,

and the unhatted quantities denote the model predictions [43]. ${ }^{6}$ The covariance matrix reads [73]

$$
(\mathbf{C})_{i j}=\left(\begin{array}{ccc}
0.0121 & 0.0129 & -0.0071 \\
0.0129 & 0.0169 & -0.0119 \\
-0.0071 & -0.0119 & 0.0121
\end{array}\right) \text {. }
$$

We then require $\chi_{\mathrm{STU}}^{2} \leq 8.025$, corresponding to a maximal $2 \sigma$ deviation given the three degrees of freedom.

\subsection{Exclusion limits from Higgs searches at LEP and LHC}

Null results from Higgs searches at collider experiments limit the signal strength of the second, non SM-like Higgs boson. Recall that its signal strength is given by the SM Higgs signal rate scaled by $(\cos \alpha)^{2}$ in the low mass region and, in the absence of Higgs-to-Higgs decays, $(\sin \alpha)^{2}$ in the high mass region. Thus, the exclusion limits can easily be translated into lower or upper limits on the mixing angle $|\sin \alpha|$, respectively. $^{7}$

\footnotetext{
6 The exact one-loop quantities from Ref. [43] render qualitatively the same constraints as the $S, T, U$ values used in Ref. [41], which were obtained from rescaled SM expressions [74].

${ }^{7}$ Here we neglect the possible influence of interference effects in the production of the light and heavy Higgs boson and its successive
}

We employ HiggsBounds-4 . 2 . 0 [52-55] to derive the exclusion limits from collider searches. The exclusion limits from the LHC experiments ${ }^{8}$ are usually given at the $95 \%$ CL. For most of the LEP results we employ the $\chi^{2}$ extension [55] of the HiggsBounds package. ${ }^{9}$ The obtained $\chi^{2}$ value will later be added to the $\chi^{2}$ contribution from the Higgs signal rates, cf. Sect. 3.6, to construct a global likelihood.

The $95 \%$ CL excluded regions of $|\sin \alpha|$ derived with HiggsBounds are shown in Fig. 4 as a function of the second Higgs mass, assuming a vanishing decay width of the Higgs-to-Higgs decay mode $H \rightarrow h h$. Since all Higgs boson production modes are reduced with respect to their SM prediction by a universal factor, limits from LHC Higgs search analyses for a SM Higgs boson can be applied straightforwardly [55]. In particular, the exclusion limits obtained from combinations of SM Higgs boson searches with various final states are highly sensitive. However, so far, such combinations have only been presented by ATLAS and CMS for the full $7 \mathrm{TeV}$ dataset $[83,84]$ and for a subset of the $8 \mathrm{TeV}$ data [85]. The strongest exclusions are therefore obtained mostly from the single search analyses of the full $7 / 8 \mathrm{TeV}$ dataset, in particular from the channel $H \rightarrow Z Z \rightarrow 4 \ell$ [86-88] in the mass region $m \in$ $[130,150] \mathrm{GeV}$ and for $m \gtrsim 190 \mathrm{GeV}$, as well as from the $H \rightarrow W W \rightarrow \ell v \ell v$ channel [10,89,90] in the mass region $m \in[160,170] \mathrm{GeV}$ due to the irreducible $Z Z$ background in the $H \rightarrow Z Z \rightarrow 4 \ell$ analyses. For Higgs masses $m \in[65,110] \mathrm{GeV}$ the only LHC exclusion limits currently available are from the ATLAS search for scalar diphoton resonances [91]. However, these constraints are weaker than the LEP limits from the channel $e^{+} e^{-} \rightarrow H Z \rightarrow(b \bar{b}) Z$ [92], as can be seen in Fig. 4b. In the remaining mass regions with $m \geq 110 \mathrm{GeV}$ the CMS limit [85] from the combination of SM Higgs analyses yields the strongest constraint. For very low Higgs masses, $m \lesssim 10 \mathrm{GeV}$, the LEP constraints come from Higgs pair production processes, $e^{+} e^{-} \rightarrow h h \rightarrow \tau^{+} \tau^{-} \tau^{+} \tau^{-}$and $\tau^{+} \tau^{-} b \bar{b}$ [92], as well as from the decay-mode independent analysis of $e^{+} e^{-} \rightarrow Z h$

\footnotetext{
Footnote 7 continued

decay. Recent studies [44,75-82] have shown that interference and finite width effect can lead to sizable deviations in the invariant mass spectra of prominent LHC search channels such as $g g \rightarrow H \rightarrow Z Z^{*} \rightarrow 4 \ell$ in the high mass region and thus should be taken into account in accurate experimental studies of the singlet-extended SM at the LHC. However, the inclusion of these effects is beyond the scope of the work presented here.

8 HiggsBounds also contains limits from the Tevatron experiments. In the singlet-extended SM, however, these limits are entirely superseded by LHC results.

9 The LEP $\chi^{2}$ information is available for Higgs masses $\geq 4 \mathrm{GeV}$. For lower masses, we take the conventional $95 \% \mathrm{CL}$ output from HiggsBounds.
} 


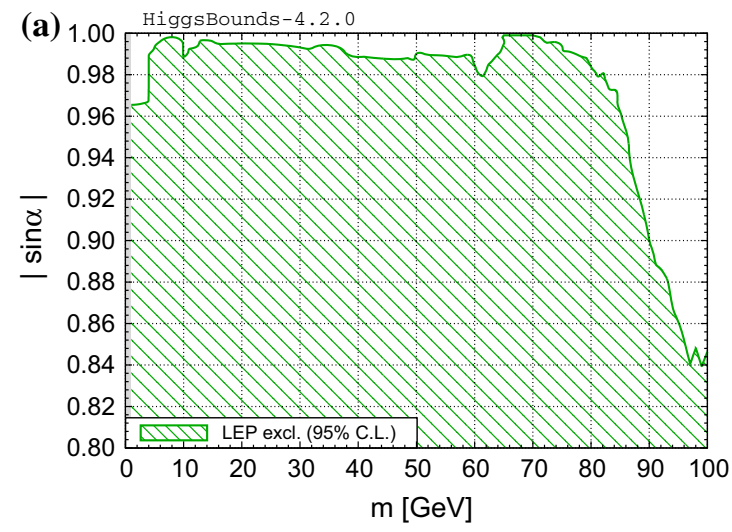

Fig. $495 \%$ CL excluded values of $|\sin \alpha|$ from LEP and LHC Higgs searches, evaluated with HiggsBounds -4.2 .0 in the mass regions $m \in[1,100] \mathrm{GeV}(\mathbf{a})$ and $m \in[100,1000] \mathrm{GeV}(\mathbf{b})$. We assume a vanishing decay width for the Higgs-to-Higgs decay mode, $\Gamma_{H \rightarrow h h}=0$,

by OPAL [93]. The latter analysis provides limits for Higgs masses as low as $1 \mathrm{keV}$.

In the presence of Higgs-to-Higgs decays, $\mathrm{BR}(H \rightarrow$ $h h) \neq 0$, additional constraints arise. In case of very low masses, $m \lesssim 3.5 \mathrm{GeV}$, these stem from the CMS search in the $H \rightarrow h h \rightarrow \mu^{+} \mu^{-} \mu^{+} \mu^{-}$channel [94], and for large masses, $m \in[260,360] \mathrm{GeV}$, from the CMS search for $H \rightarrow h h$ with multileptons and photons in the final state [95]. These limits will be discussed separately in Sect. 4. Note that the limit from SM Higgs boson searches in the mass range $m \gtrsim 250 \mathrm{GeV}$, as presented in Fig. 4b, will diminish in case of non-vanishing $\operatorname{BR}(H \rightarrow h h)$ due to a suppression of the SM Higgs decay modes. We find in the full scan (see Sect. 4) that, in general, $\mathrm{BR}(H \rightarrow h h)$ can be as large as $\sim 70 \%$ in this model. Neglecting the correlation between $\sin \alpha$ and $\operatorname{BR}(H \rightarrow h h)$ for a moment, such large branching fractions could lead to a reduction of the upper limit on $|\sin \alpha|$ obtained from SM Higgs searches by a factor of $\sim 1 / \sqrt{1-\mathrm{BR}(H \rightarrow h h)} \lesssim 1.8$. However, once all other constraints (in particular from the NLO calculation of $\left.m_{W}\right)$ are taken into account, only $\mathrm{BR}(H \rightarrow h h)$ values of up to $40 \%$ are found; see Sect. 4.1, Fig. 11b. Moreover, in the mass region $m_{H} \sim 270-360 \mathrm{GeV}$ where the largest values of $\operatorname{BR}(H \rightarrow h h)$ appear, the $m_{W}$ constraint on $|\sin \alpha|$ is typically stronger than the constraints from SM Higgs searches, even if $\operatorname{BR}(H \rightarrow h h)=0$ is assumed in the latter. Therefore, given the present Higgs search exclusion limits, the signal rate reduction currently does not have a visible impact on the viable parameter space..$^{10}$

\footnotetext{
10 Note, however, that this may change in future with significantly improved exclusion limits from SM Higgs searches.
}

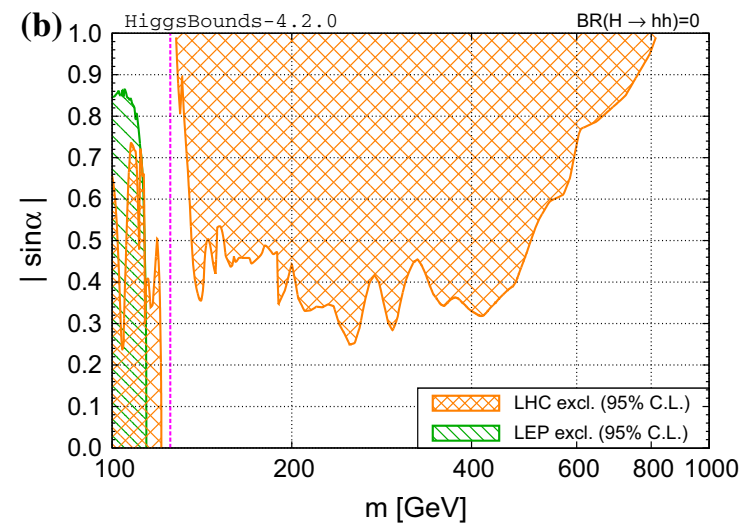

hence the displayed results in the high mass region correspond to the most stringent upper limit on $|\sin \alpha|$ that can be obtained from current LHC Higgs searches. The other Higgs boson mass is set to $125.14 \mathrm{GeV}$ and is indicated by the dashed, magenta line in $\mathbf{b}$

\subsection{Higgs boson signal rates measured at the LHC}

The compatibility of the predicted signal rates for the Higgs state at $\sim 125 \mathrm{GeV}$ with the latest measurements from ATLAS [4-6,96,97] and CMS [7-10] is evaluated with HiggsSignals -1.3 .0 by means of a statistical $\chi^{2}$ measure. The implemented observables are listed in Table 1 . In the following we denote this $\chi^{2}$ value by $\chi_{\mathrm{HS}}^{2}$, which also includes the $\chi^{2}$ contribution from the Higgs mass observables evaluated within HiggsSignals. The latter, however, only yields non-trivial constraints on the parameter space if the fit allows a varying Higgs mass in the vicinity of $125 \mathrm{GeV}$. In the low mass scenario, where one of the Higgs bosons is within the kinematical range of the LEP experiment, the $\chi^{2}$ value obtained from the

Table 1 Higgs boson signal rate and mass observables from the LHC experiments, as implemented in Higgs Signals-1.3.0 and used in this analysis. For the mass measurements we combined the systematic and statistical uncertainty in quadrature

\begin{tabular}{llll}
\hline Experiment & Channel & $\begin{array}{l}\text { Obs. signal } \\
\text { rate }\end{array}$ & $\begin{array}{l}\text { Obs. mass } \\
(\mathrm{GeV})\end{array}$ \\
\hline ATLAS & $h \rightarrow W W \rightarrow \ell \nu \ell v[96]$ & $1.08_{-0.20}^{+0.22}$ & - \\
ATLAS & $h \rightarrow Z Z \rightarrow 4 \ell[5]$ & $1.44_{-0.33}^{+0.40}$ & $124.51 \pm 0.52$ \\
ATLAS & $h \rightarrow \gamma \gamma[4]$ & $1.17_{-0.27}^{+0.27}$ & $125.98 \pm 0.50$ \\
ATLAS & $h \rightarrow \tau \tau[97]$ & $1.42_{-0.37}^{+0.43}$ & - \\
ATLAS & $V h \rightarrow V(b \bar{b})[6]$ & $0.51_{-0.37}^{+0.40}$ & - \\
CMS & $h \rightarrow W W \rightarrow \ell \nu \ell v[10]$ & $0.72_{-0.18}^{+0.20}$ & - \\
CMS & $h \rightarrow Z Z \rightarrow 4 \ell[9]$ & $0.93_{-0.25}^{+0.29}$ & $125.63 \pm 0.45$ \\
CMS & $h \rightarrow \gamma \gamma[7]$ & $1.14_{-0.23}^{+0.26}$ & $124.70 \pm 0.34$ \\
CMS & $h \rightarrow \tau \tau[8]$ & $0.78_{-0.27}^{+0.27}$ & - \\
CMS & $V h \rightarrow V(b \bar{b})[8]$ & $1.00_{-0.50}^{+0.50}$ & - \\
\hline
\end{tabular}




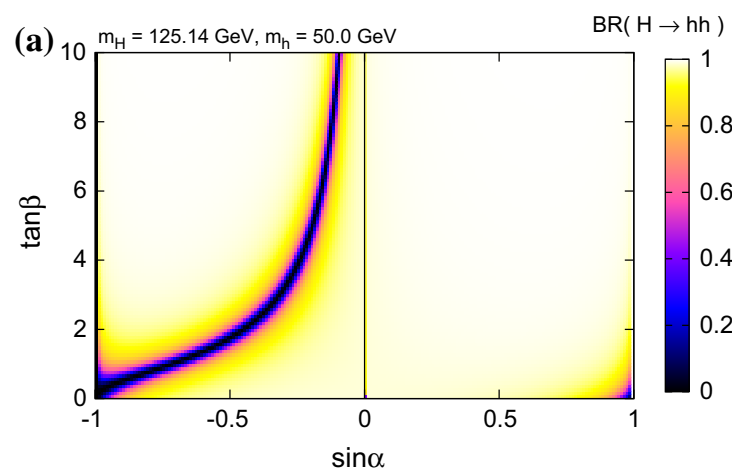

Fig. 5 Branching ratio $\operatorname{BR}(H \rightarrow h h)$ in the $(\sin \alpha, \tan \beta)$ plane for fixed Higgs masses $m_{h}=50 \mathrm{GeV}$ and $m_{H}=125.14 \mathrm{GeV}$. It becomes minimal for either $\sin \alpha=0, \cos \alpha=0$ or $\tan \beta=-\cos \alpha / \sin \alpha$. a $(\sin \alpha, \tan \beta)$ plane. b Zoomed region of the $(\sin \alpha, \tan \beta)$ plane. The

HiggsBounds LEP $\chi^{2}$ extension, denoted as $\chi_{\text {LEP }}^{2}$, is added to the HiggsSignals $\chi^{2}$ to construct the global likelihood

$\chi_{\mathrm{tot}}^{2}=\chi_{\mathrm{HS}}^{2}+\chi_{\mathrm{LEP}}^{2}$.

The 68 and $95 \%$ CL parameter regions of the model are approximated by the $\chi^{2}$ difference to the minimal $\chi^{2}$ value found at the best-fit point, $\Delta \chi^{2}=\chi^{2}-\chi_{\min }^{2}$, taking on values of 1 (2.30) and 4 (6.18) in the case of a 1 (2)-dimensional projected parameter space, respectively.

When both Higgs masses are fixed, the fit depends on two free parameters, namely $\sin \alpha$ and $\tan \beta$. The latter can only influence the signal rates of the Higgs boson $H$ if the additional decay mode $H \rightarrow h h$ is accessible. The branching fraction $\operatorname{BR}(H \rightarrow h h)$ then leads to a decrease of all other decay modes and hence to a reduction of the predictions for the measured signal rates, cf. Eq. (16). The sensitivity to $\tan \beta$ via the signal rate measurements is thus only given if the heavier Higgs state is interpreted as the discovered particle, $m_{H} \sim 125 \mathrm{GeV}$ (low mass region), and the second Higgs state is sufficiently light, $m_{h} \lesssim 62 \mathrm{GeV}$. If the $H \rightarrow h h$ decay is not kinematically accessible, or in the case where the light Higgs is considered as the discovered Higgs state at $\sim 125 \mathrm{GeV}$, there are no relevant experimental constraints on $\tan \beta$.

In the low mass region, the Higgs signal rate measurements constrain the modulus of the mixing angle $\sin \alpha$ to be close to 1, such that the heavy Higgs boson has nearly the same coupling strengths as the SM Higgs boson. Moreover, in order to obtain sizable predictions for the measured signal rates, the branching ratio $\mathrm{BR}(H \rightarrow h h)$ must not be too large. We illustrate its dependence on $\tan \beta$ in Fig. 5, where we exemplarily show the branching ratio $\mathrm{BR}(H \rightarrow h h)$ in the $(\sin \alpha, \tan \beta)$ plane for fixed Higgs boson masses of $m_{h}=50 \mathrm{GeV}$ and $m_{H}=125.14 \mathrm{GeV}$. As can be seen in

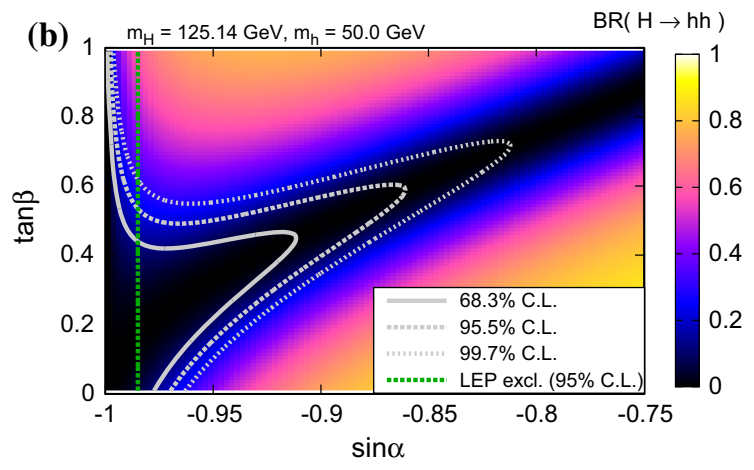

gray contours indicate the 1,2 and $3 \sigma$ regions preferred by the signal rates; the green, dashed line displays the $95 \%$ CL limit from LEP, cf. Sect. 3.5

Fig. 5a, the decay $H \rightarrow h h$ is dominant over large regions of the parameter space, with the exception of three distinct cases: The branching ratio $\mathrm{BR}(H \rightarrow h h)$ exactly vanishes in the case that

(i) $\sin \alpha=0$, (ii) $\cos \alpha=0$ or

(iii) $\tan \beta=-\cos \alpha / \sin \alpha$.

In the first case (i) all couplings of the heavy Higgs boson to SM particles vanish completely, thus this case is highly excluded by observations. The second case (ii) corresponds to the complete decoupling of the lighter Higgs boson, such that the heavier Higgs is identical to the SM Higgs boson. In the third and more interesting case (iii) the branching fraction can be expanded in powers of $(\tan \beta+\cos \alpha / \sin \alpha)$ :

$$
\begin{aligned}
& \mathrm{BR}(H \rightarrow h h)=\frac{\sqrt{1-\frac{4 m_{h}^{2}}{m_{H}^{2}}}\left(m_{h}^{2}+\frac{m_{H}^{2}}{2}\right)^{2}}{8 \pi v^{2} m_{H} \Gamma_{\mathrm{SM}, \operatorname{tot}}\left(m_{H}\right)} \cos ^{2} \alpha \sin ^{4} \alpha \\
& \times\left(\tan \beta+\frac{\cos \alpha}{\sin \alpha}\right)^{2}+\mathcal{O}\left[\left(\tan \beta+\frac{\cos \alpha}{\sin \alpha}\right)^{3}\right],
\end{aligned}
$$

where $\Gamma_{\mathrm{SM}}$, tot $\left(m_{H}\right)$ is the total width in the SM for a Higgs boson at mass $m_{H}$.

In Fig. $5 \mathrm{~b}$ we show a zoom of the $(\sin \alpha, \tan \beta)$ plane, focusing on the low-BR $(H \rightarrow h h)$ valley and $\sin \alpha$ values close to -1 . We furthermore indicate the parameter regions which are allowed at the 1,2 , and $3 \sigma$ level by the Higgs signal rate measurements by the gray contour lines. The maximally values of $\mathrm{BR}(H \rightarrow h h) \approx 26 \%$ allowed by the Higgs signal rate measurements at $95 \% \mathrm{CL}$ are found for $\sin \alpha$ very close to -1 and large $\tan \beta$ values, i.e. in the vicinity of case (ii) discussed above. In the given example with $m_{h}=50 \mathrm{GeV}$, the $95 \% \mathrm{CL}$ exclusion from LEP searches, as discussed in Sect. 3.5 (cf. Fig. 4a), imposes $\sin \alpha \lesssim-0.985$ and is indicated in Fig. $5 b$ by the green, dashed line. 


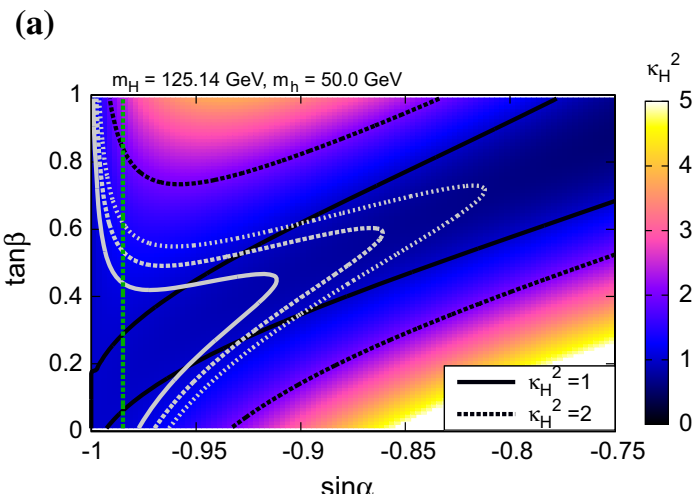

Fig. 6 Total width scale factor $\kappa_{H}^{2}=\Gamma_{\text {tot }} / \Gamma_{\text {tot,SM }}$ for fixed Higgs masses $m_{h}=50 \mathrm{GeV}$ and $m_{H}=125.14 \mathrm{GeV}: \mathbf{a}(\sin \alpha, \tan \beta)$ plane. The black solid and dashed line indicate constant values of $K_{H}^{2}=1$

Finally, we plot the total width scaling factor, defined by $\kappa_{H}^{2}=\Gamma_{\text {tot }} / \Gamma_{\text {tot, SM }}$, in the $(\sin \alpha, \tan \beta)$ plane in Fig. 6 a. We furthermore plot $\kappa_{H}^{2}$ for the parameter regions favored by the Higgs signal rates in Fig. 6b. The largest values of $\kappa_{H}^{2}$ allowed by both the signal rates and the LEP constraints at $95 \% \mathrm{CL}$ are obtained for $\sin \alpha$ close to -1 . In the example of $m_{h}=50 \mathrm{GeV}$ discussed here, the total width is increased by up to around $34 \%$ with respect to the SM. This maximal value of total width enhancement is independent of the light Higgs mass (assuming that the channel $H \rightarrow h h$ is kinematically accessible).

We now want to draw the attention to the intermediate mass range, where both mass eigenstates can contribute to the signal strength measurements at the LHC. If the masses of the two Higgs bosons are well separated, the signal yields measured in the LHC Higgs analyses can be assumed to be solely due to the one Higgs boson lying in the vicinity of the signal, $m \sim 125 \mathrm{GeV}$. However, in analyses with a poor mass resolution, as is typically the case in search analyses for the decay modes $H \rightarrow W^{+} W^{-}, H \rightarrow \tau^{+} \tau^{-}$, and $V H \rightarrow b \bar{b}$, the signal contamination from the second Higgs boson needs to be taken into account if its mass is not too far away from $125 \mathrm{GeV}$. While a proper treatment of this case can only be done by the experimental analyses, Higgs Signals employs a Higgs boson assignment procedure to approximately account for this situation [56]. Based on the experimental mass resolution of the analysis and the difference between the predicted mass and the mass position where the measurement has been performed, HiggsSignals decides whether the signal rates of multiple Higgs states need to be combined. Hence, superpositions of the two Higgs signal rates considered here are possible if the second Higgs mass lies within $100 \mathrm{GeV} \lesssim m \lesssim$ $150 \mathrm{GeV}$.

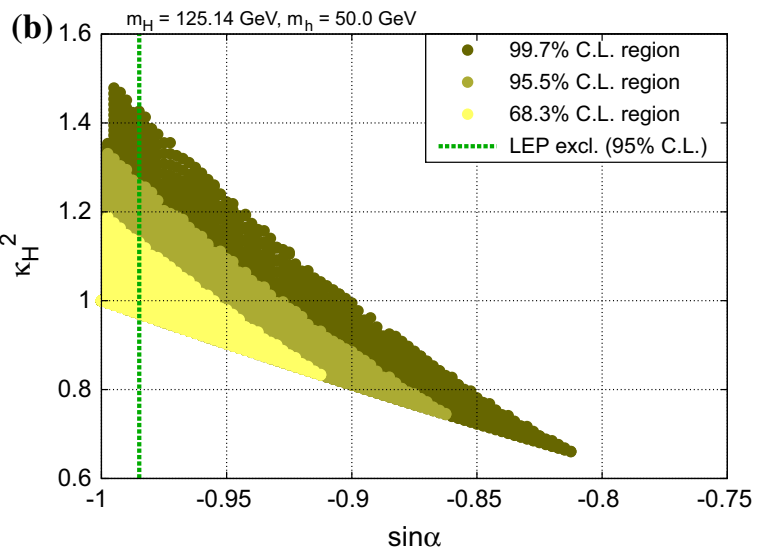

and 2, respectively. The other contour lines are the same as in Fig. 5b. b Total width scale factor $K_{H}^{2}$ for the $1 \sigma, 2 \sigma$ and $3 \sigma$ regions favored by the Higgs boson signal rates

In Fig. 7 we show the HiggsSignals $\Delta \chi^{2}$ value obtained from the signal rate observables as a function of the second Higgs boson mass $m$ and the mixing angle $\sin \alpha$. The mass of the other Higgs boson is fixed at $m=125.14 \mathrm{GeV}$. The scan range for $m$ extends over both the low mass and high mass region. Since the Higgs boson at $\sim 125 \mathrm{GeV}$ needs sufficiently large signal rates to accommodate for the observed SM-like Higgs signal strength, small (large) values of $\sin \alpha$ are favored in the high (low) mass region, such that the second Higgs boson is rather decoupled. We furthermore show the parameter space excluded at $95 \%$ CL by LEP and LHC searches, as previously discussed in Sect. 3.5 in Fig. 4.

In the case of nearly mass-degenerate Higgs bosons, $m_{h} \approx m_{H}=125.14 \mathrm{GeV}$, the sensitivity on the mixing angle $\sin \alpha$ significantly decreases, as the signal rates of the two Higgs states are always superimposed. There remains a slight dependence of the total signal rate on the Higgs masses, though, since the production cross sections and branching ratios are mass dependent. Moreover, depending on the actual mass splitting and mixing angle, potential effects may possibly be seen in the invariant mass distributions of the high-resolution LHC channels $p p \rightarrow H \rightarrow \gamma \gamma$ [7] and $p p \rightarrow H \rightarrow Z Z^{*} \rightarrow 4 \ell$, at a future linear collider like the ILC $[18,42]$ or eventually a muon collider $[42,98]$. However, the sensitivity on $\sin \alpha$ completely vanishes in the case of exact mass degeneracy, $m_{h}=m_{H}$, such that the singlet-extended SM becomes indistinguishable from the SM.

The weak $\Delta \chi^{2}$ dependence on $m$ outside of the massdegenerate region, i.e. for $m \gtrsim 128 \mathrm{GeV}$ and $m \lesssim 122 \mathrm{GeV}$, is caused by the superposition of the signal rates of both Higgs bosons in some of the $H \rightarrow W^{+} W^{-}, \tau^{+} \tau^{-}$, and $b \bar{b}$ channels, as discussed above. These structures depend on 
Fig. $7 \Delta \chi^{2}$ distribution from the Higgs signal rate observables, obtained from HiggsSignals-1.3.0, as a function of the second Higgs boson mass $m$ and the mixing angle $\sin \alpha$. The mass of the other Higgs boson is fixed at $m=125.14 \mathrm{GeV}$, indicated by the dashed, magenta line. The gray contour lines indicate the favored parameter space at 68.3 , 95.5, and $99.7 \%$ CL, solely based on the Higgs signal rate observables. The green striped (orange patterned) region is excluded by LEP (LHC) Higgs searches at $95 \%$ CL, cf. also Fig. 4. For Higgs masses $m$ below $100 \mathrm{GeV}$ and beyond around $152 \mathrm{GeV}$ the signal rate constraints are independent of $m$

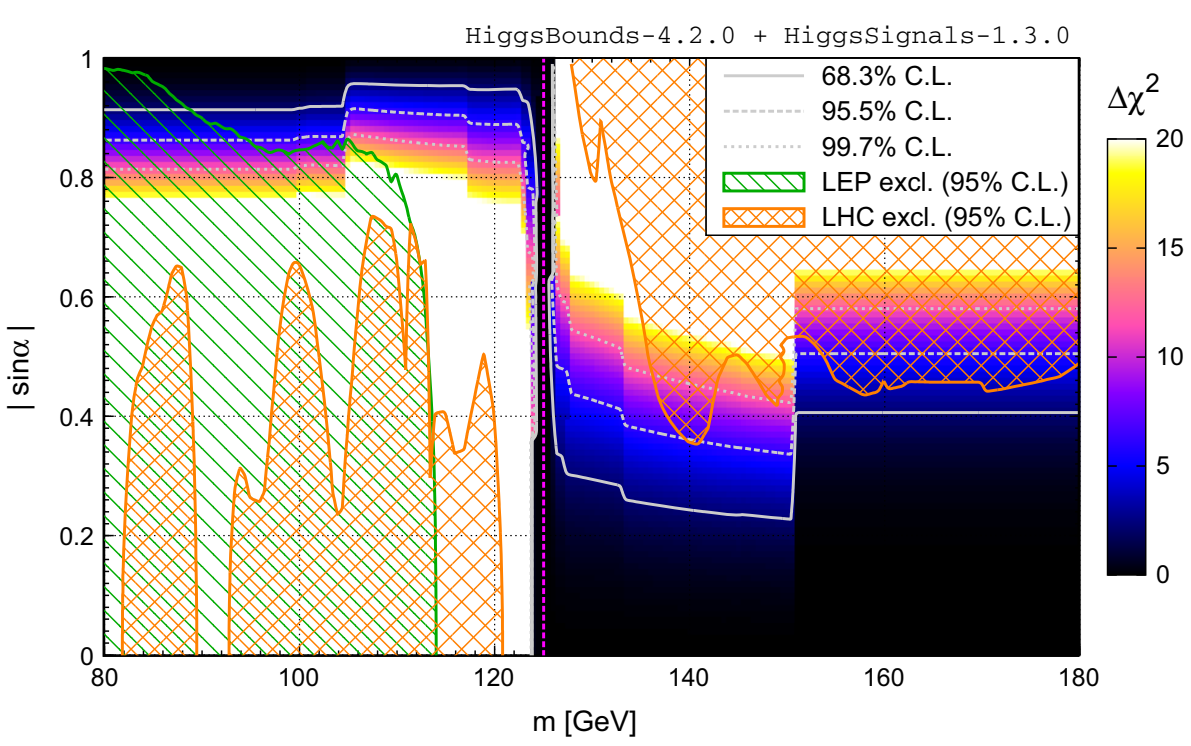

the details of the implementation within HiggsSignals, in particular on the assumed experimental resolution for each analysis. For Higgs masses $m$ below $100 \mathrm{GeV}$ and beyond around $152 \mathrm{GeV}$ the $\sin \alpha$ limit from the signal rates is independent ${ }^{11}$ of $m$.

We see that for Higgs masses $m$ in the range between $\sim 100$ and $150 \mathrm{GeV}$, the constraints from the Higgs signal rates are more restrictive than the exclusion limits from Higgs searches at LEP and LHC. For lower Higgs masses, $m<$ $100 \mathrm{GeV}$, the LEP limits (cf. Fig. 4a) generally yield stronger constraints on the parameter space. For higher Higgs masses, $m \in[150,500] \mathrm{GeV}$, the direct LHC limits (cf. Fig. 4b) are slightly stronger than the constraints from the signal rates, however, this picture reverses again for Higgs masses beyond $500 \mathrm{GeV}$, where direct heavy Higgs searches become less sensitive.

\section{Results of the full parameter scan}

In this section we investigate the interplay of all theoretical and experimental constraints discussed in the previous section on the real singlet-extended SM parameter space, specified by

$m \equiv m_{h / H}, \sin \alpha, \tan \beta$

We separate the discussion into the high mass, the low mass and the intermediate (or degenerate) mass region of the parameter space. In the high and low mass region, we keep one of the Higgs masses fixed at $125.14 \mathrm{GeV}$ and vary

\footnotetext{
11 This statement is only true if the Higgs state at $\sim 125 \mathrm{GeV}$ does not decay to the lighter Higgs. As discussed above, at low light Higgs masses $m_{h}<m_{H} / 2$, the branching ratio $\mathrm{BR}(H \rightarrow h h)$ can reduce the signal rates of the heavy Higgs decaying to SM particles.
}

the other, while in the intermediate mass region we treat both Higgs masses as scan parameters. In the following we first present results for fixed mass $m$ in order to facilitate the understanding of the respective parameter space in dependence of $\sin \alpha, \tan \beta$. These discussions will then be extended by a more general scan, where all parameters are allowed to vary simultaneously. For each of these scans, we generate around $\mathcal{O}\left(10^{5}-10^{6}\right)$ points. We close the discussion of each mass region by commenting on the relevant collider phenomenology.

\subsection{High mass region}

In this section, we explore the parameter space of the high mass region, $m \in[130,1000] \mathrm{GeV}$. In general, for masses $m \geq 600 \mathrm{GeV}$, our results agree with those presented in Ref. [41]. However, we obtain stronger bounds on the maximally allowed value of $|\sin \alpha|$ due to the constraints from the NLO calculation of $m_{W}$ [43], which has not been available for the previous analysis [41]. As has been discussed in Sect. 3.4, Fig. 3, the constraints from $m_{W}$ are much more stringent than those obtained from the oblique parameters $S$, $T$, and $U$ in the high mass region.

We compile all previously discussed constraints on the maximal mixing angle in Fig. 8. Furthermore, the (onedimensional) allowed regions in $|\sin \alpha|$ and $\tan \beta$ are given in Table 2 for fixed values of $m .{ }^{12}$ Here, the allowed range of $|\sin \alpha|$ is evaluated for fixed $\tan \beta=0.15$ and we explicitly specify the relevant constraint that provides in the upper limit on $|\sin \alpha|$. We find the following generic features: for Higgs

\footnotetext{
12 Note that the upper limit on $|\sin \alpha|$ from the Higgs signal rates is based on a two-dimensional $\Delta \chi^{2}$ profile (for floating $m_{h}$ ) in Fig. 8, whereas in Table 2 the one-dimensional $\Delta \chi^{2}$ profile (for fixed $m_{h}$ ) is used. This leads to small differences in the obtained limit.
} 


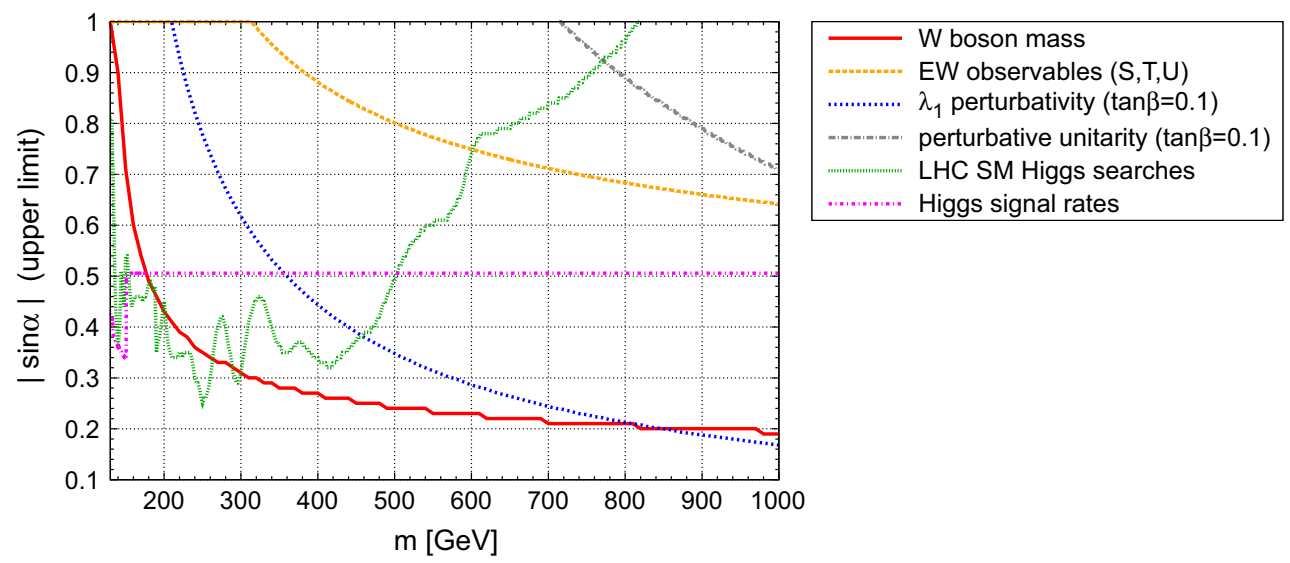

Fig. 8 Comparison of all constraints on $|\sin \alpha|$ as a function of the heavy Higgs mass $m$ in the high mass region. The $\lambda_{1}$ perturbativity and perturbative unitarity constraint have been evaluated for $\tan \beta=0.1$

Table 2 Allowed ranges for $\sin \alpha$ and $\tan \beta$ in the high mass region for fixed Higgs masses $m$. The allowed interval of $\sin \alpha$ was determined at $\tan \beta=0.15$. The $95 \%$ CL limits on $\sin \alpha$ from the Higgs signal rates are derived from one-dimensional fits and taken at $\Delta \chi^{2}=4$. The lower limit on $\sin \alpha$ always stems from vacuum stability, and the upper limit on $\tan \beta$ always from perturbativity of $\lambda_{2}$, evaluated at $\sin \alpha=0.1$. The source of the most stringent upper limit on $\sin \alpha$ is named in the third column. We fixed $m_{h}=125.14 \mathrm{GeV}$, and the stability and perturbativity were tested at a scale of $\sim 4 \times 10^{10} \mathrm{GeV}$

\begin{tabular}{llll}
\hline$m(\mathrm{GeV})$ & $|\sin \alpha|$ & Source upper limit & $(\tan \beta)_{\max }$ \\
\hline 1000 & {$[0.018,0.17]$} & $\lambda_{1}$ perturbativity & 0.23 \\
900 & {$[0.022,0.19]$} & $\lambda_{1}$ perturbativity & 0.26 \\
800 & {$[0.027,0.21]$} & $m_{W}$ at NLO & 0.29 \\
700 & {$[0.031,0.21]$} & $m_{W}$ at NLO & 0.33 \\
600 & {$[0.038,0.23]$} & $m_{W}$ at NLO & 0.39 \\
500 & {$[0.046,0.24]$} & $m_{W}$ at NLO & 0.47 \\
400 & {$[0.055,0.27]$} & $m_{W}$ at NLO & 0.59 \\
300 & {$[0.067,0.31]$} & $m_{W}$ at NLO & 0.78 \\
200 & {$[0.090,0.43]$} & $m_{W}$ at NLO & 1.17 \\
180 & {$[0.10,0.46]$} & Signal rates & 1.30 \\
160 & {$[0.11,0.46]$} & Signal rates & 1.46 \\
140 & {$[0.16,0.31]$} & Signal rates & 1.67 \\
\hline
\end{tabular}

masses $m \gtrsim 200-300 \mathrm{GeV}$, the $W$ boson mass NLO calculation provides the upper limit on $|\sin \alpha|$, at lower masses the LHC constraints at $95 \%$ CL from direct Higgs searches and the signal rate measurements are most relevant. The purely theory-based limits from perturbativity of $\lambda_{1}$ only become important for $m \gtrsim 800 \mathrm{GeV}$. Furthermore, in the whole mass range, the minimal value of $|\sin \alpha|$ and the maximal value of $\tan \beta$ are determined by vacuum stability and perturbativity of the couplings.

The corresponding (two-dimensional) allowed regions in the $(\sin \alpha, \tan \beta)$ plane for fixed Higgs masses are shown in Fig. 9. Their shapes are largely dictated by the perturbativ- ity and vacuum stability requirements of the RGE evolved couplings, thus basically resembling the features observed before in Fig. 2 for $m_{H}=600 \mathrm{GeV}$. Here, however, the maximally allowed values for the mixing angle $\sin \alpha$ stem now from the NLO calculation of $m_{W}$ or, at rather low masses $m_{H} \lesssim 200 \mathrm{GeV}$, from the Higgs signal rates and/or exclusion limits. In all cases, the upper limit on $\tan \beta$ stems from the perturbativity requirement of RGE evolved couplings. For the degenerate case, $m_{h} \approx m_{H} \approx 125 \mathrm{GeV}$, we a priori find no upper or lower limit on the mixing angle. In the degenerate case we do not take limits from RGE running into account, hence the only constraint stems from perturbative unitarity which renders an upper limit on $\tan \beta$.

We now extend the discussion and treat $m_{H}$ as a free model parameter. The results are presented as scatter plots using the following color scheme:

- light gray points include all scan points which are not further classified,

- dark gray points fulfill constraints from perturbative unitarity, perturbativity of the couplings, RGE running and the $W$ boson mass, as discussed in Sects. 3.1-3.4,

- blue points additionally pass the $95 \%$ CL exclusion limits from Higgs searches,

- red/yellow points fulfill all criteria above and furthermore lie within a $1 / 2 \sigma$ regime favored by the Higgs signal rate observables.

The results are presented in Fig. 10 in terms of twodimensional scatter plots in the three scan parameters. The point distribution in the $(\sin \alpha, \tan \beta)$ plane shown in Fig. 10a neatly resembles the features of Fig. 9 discussed above: Small mixings are forbidden from the requirement of vacuum stability, while the maximal value for the mixing angle, $|\sin \alpha| \lesssim 0.50$, is limited by the Higgs signal rate observables. Figure 10b illustrates how the upper limit on $\tan \beta$, 


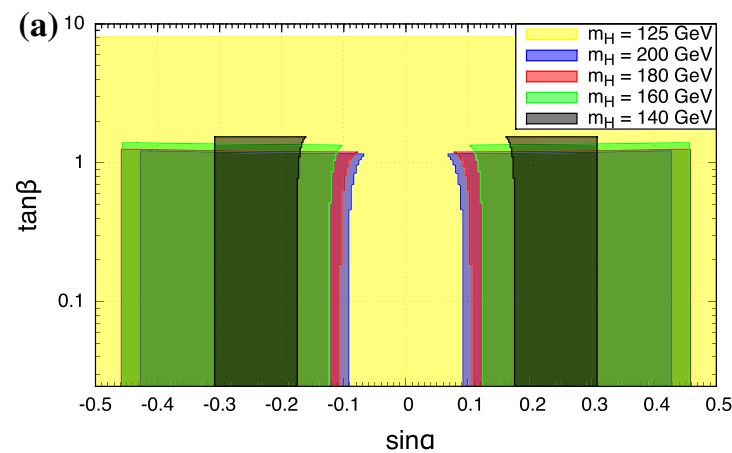

Fig. 9 Allowed regions in the $(\sin \alpha, \tan \beta)$ plane in the high mass region for fixed Higgs masses $m$. For $m_{H} \gtrsim 200 \mathrm{GeV}$, the upper limit on the mixing angle stems from $m_{W}$, while for $m_{H} \lesssim 200 \mathrm{GeV}$ the

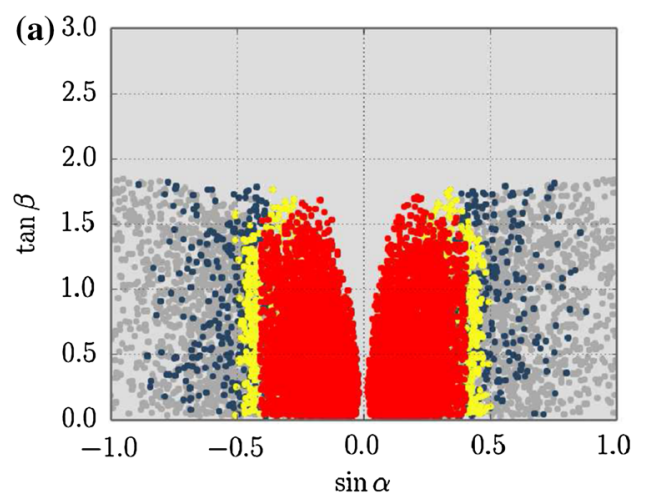

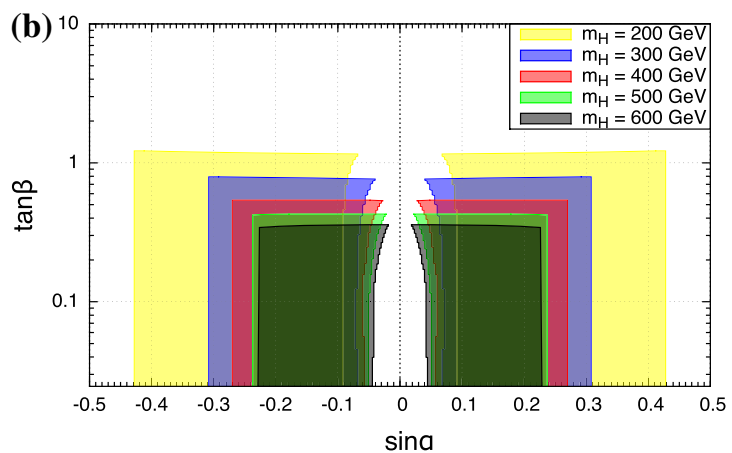

upper limit is given by the signal strength measurements as well as experimental searches (cf. also Fig. 8). a Higgs masses below $200 \mathrm{GeV}$. b Higgs masses above $200 \mathrm{GeV}$

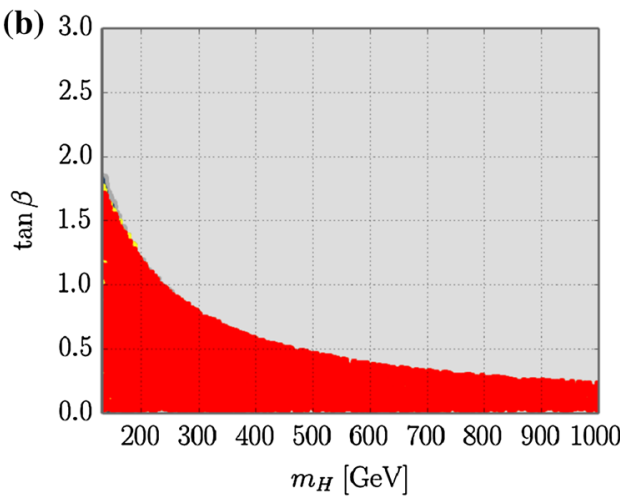

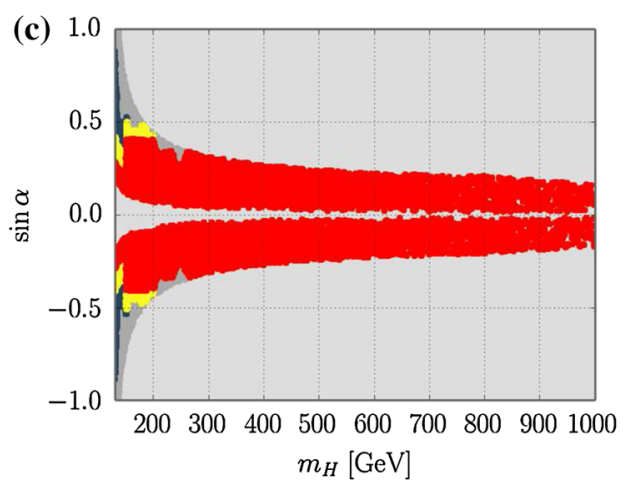

Fig. 10 Two-dimensional parameter correlations between $m_{H}, \tan \beta$, and $\sin \alpha$ in the high mass region. See text for a description of the color coding. a $(\sin \alpha, \tan \beta)$ plane. $\mathbf{b}\left(m_{H}, \tan \beta\right)$ plane. $\mathbf{c}\left(m_{H}, \sin \alpha\right)$ plane

which stems from the perturbativity requirement of $\lambda_{2}$, roughly follows the expected $\sim m_{H}^{-1}$ scaling, cf. Eq. (22). Finally, we can easily recognize the upper limit on the mixing angle $\sin \alpha$ from the $m_{W}$ constraint and the perturbativity requirement of $\lambda_{1}$, cf. Fig. 3 , in the point distribution in the $\left(m_{H}, \sin \alpha\right)$ plane shown in Fig. 10c. These constraints provide the most stringent upper limit on $|\sin \alpha|$ for Higgs masses $m_{H} \gtrsim 260 \mathrm{GeV}$. At lower Higgs masses, the upper limit is set by the Higgs signal rate measurements and exclusion limits from Higgs searches at the LHC, cf. Fig. 8. Here it is interesting to see that the favored $|\sin \alpha|$ region at Higgs masses $m_{H}$ between 130 and $\sim 152 \mathrm{GeV}$ is more restricted than at higher Higgs masses. Two effects play a role here: Firstly, the lower limit on $|\sin \alpha|$ from the vacuum stability requirement is stronger than at larger Higgs masses; And secondly, the heavy Higgs boson lies still in the vicinity of the discovered Higgs state, such that their signal rates are combined in the $H \rightarrow \tau \tau, H \rightarrow W W$, and $V H \rightarrow V(b \bar{b})$ channels, where the mass resolution is poor. In total, these channels, however, favor a slightly lower signal strength than obtained for a SM Higgs, thus the fit slightly prefers larger Higgs masses $m_{H}$, where the signal rates are not added 

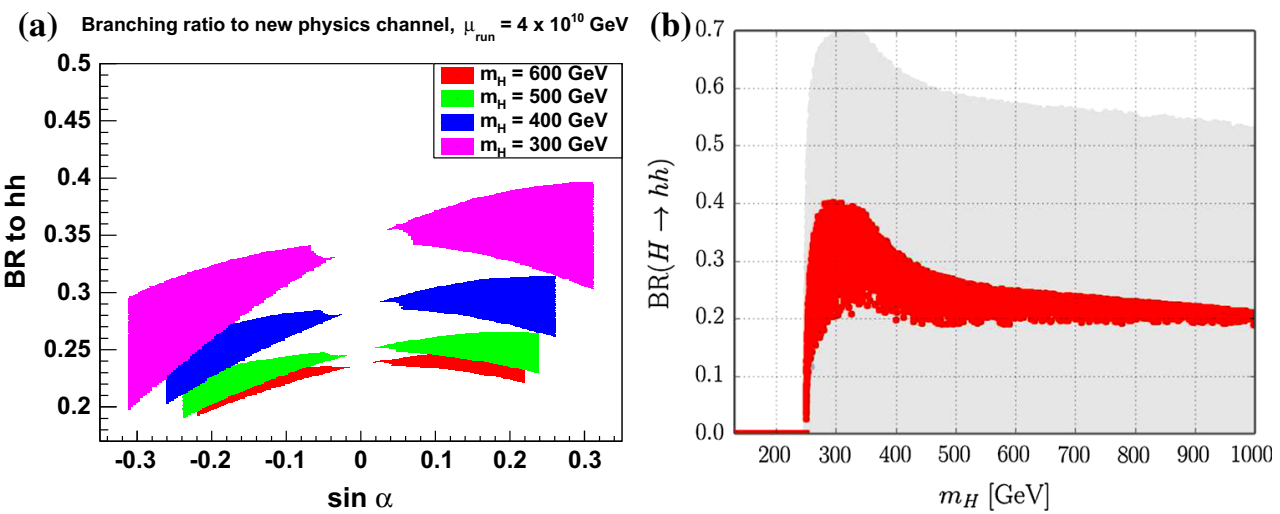

Fig. 11 Allowed branching ratios of the Higgs-to-Higgs decay channel $H \rightarrow h h$ in the high mass scenario. a Allowed values of BR $(H \rightarrow h h$ ) as function of $\sin \alpha$ for fixed Higgs masses $m_{H}$. b BR $(H \rightarrow h h)$ as a function of $m_{H}$, using the same color code as in Fig. 10

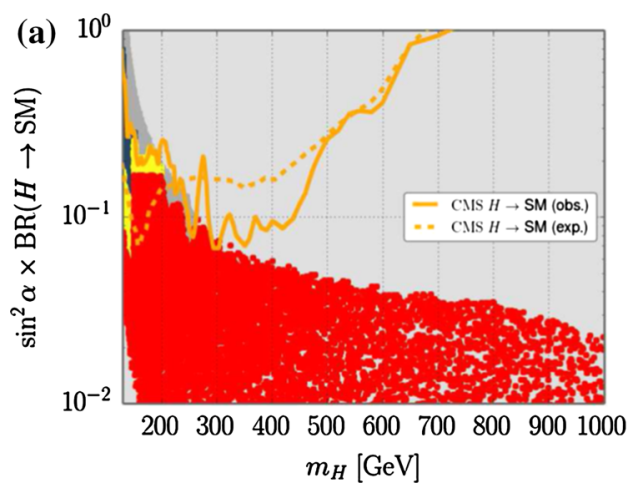

Fig. 12 Collider signal rates of the heavy Higgs boson $H$ decaying into SM particles (a) or into two light Higgs bosons, $H \rightarrow h h(\mathbf{b})$, in dependence of the heavy Higgs mass, $m_{H}$. The rates are normalized to the inclusive SM Higgs production cross section at the corresponding mass value [35,101,102]. a Heavy Higgs signal rate with SM particles in the final state. The orange solid (dashed) curves indicate the

for these observables within HiggsSignals, cf. also Fig. 7.

We now turn to the discussion of the collider phenomenology of the high mass region. Experimentally, the model can be probed by searches for a SM-like Higgs boson with a reduced signal rate and total decay widths, or by direct searches for the Higgs-to-Higgs decay mode $H \rightarrow h h$, where $h$ is the light Higgs boson at around $125 \mathrm{GeV}$.

We show the allowed values of the branching ratio $\mathrm{BR}(H \rightarrow h h)$, given by Eq. (16), in Fig. 11. In Fig. 11a we show the dependence on $\sin \alpha$ exemplarily for fixed Higgs masses $m_{H}$, whereas the full $m_{H}$ dependence is displayed in Fig. 11b, using the same color code as above. We observe that the maximal values of $\mathrm{BR}(H \rightarrow h h)$ are $\sim 40 \%$, reached for large, positive $\sin \alpha$ values [41], and low Higgs masses $m \sim 300 \mathrm{GeV}$. At higher Higgs masses the branching ratio $\mathrm{BR}(H \rightarrow h h)$ is around $20 \%$ or slightly higher.

The LHC production cross section of the heavier Higgs boson $H$ is given by the SM Higgs production cross section

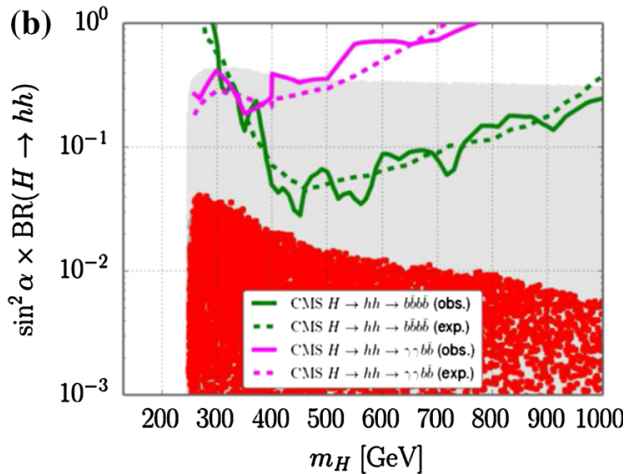

observed (expected) $95 \%$ CL limits from the latest CMS combination of SM Higgs searches [85]. b Heavy Higgs signal rate with light Higgs bosons in the final state. We display the current expected and observed $95 \%$ CL limits from CMS $H \rightarrow h h$ searches with $\gamma \gamma b \bar{b}$ [99] and $b \bar{b} b \bar{b}$ [100]

multiplied by $\sin ^{2} \alpha$. For convenience, we introduce the rate scale factors [41]

$$
\begin{gathered}
\kappa \equiv \frac{\sigma}{\sigma_{\mathrm{SM}}} \times \mathrm{BR}(H \rightarrow \mathrm{SM})=\sin ^{4} \alpha \frac{\Gamma_{\mathrm{SM}, \mathrm{tot}}}{\Gamma_{\mathrm{tot}}}, \\
\kappa^{\prime} \equiv \frac{\sigma}{\sigma_{\mathrm{SM}}} \times \mathrm{BR}(H \rightarrow h h)=\sin ^{2} \alpha \frac{\Gamma_{H \rightarrow h h}}{\Gamma_{\mathrm{tot}}},
\end{gathered}
$$

for the heavy Higgs collider processes leading to SM particles or two light Higgs bosons in the final state, respectively. Here, $\mathrm{BR}(H \rightarrow \mathrm{SM})$ comprises all possible Higgs decay modes to SM particles. Note that $\kappa+\kappa^{\prime}=\sin ^{2} \alpha$ corresponds to the inclusive heavy Higgs production rate, normalized to the inclusive SM Higgs production rate $[35,101,102]$.

The predicted signal rates normalized to the SM production cross section, Eqs. (32) and (33), are shown as a function of the Higgs mass $m_{H}$ for the high mass region in Fig. 12. We furthermore display the current $95 \%$ CL exclusion limits from the latest CMS combination of SM Higgs searches [85], 


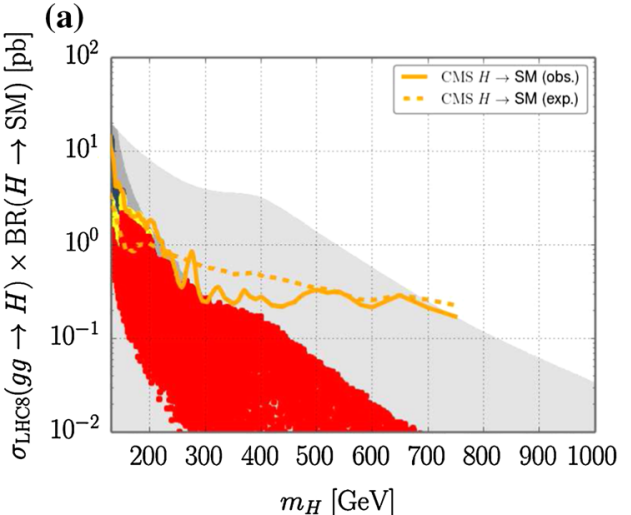

(c)

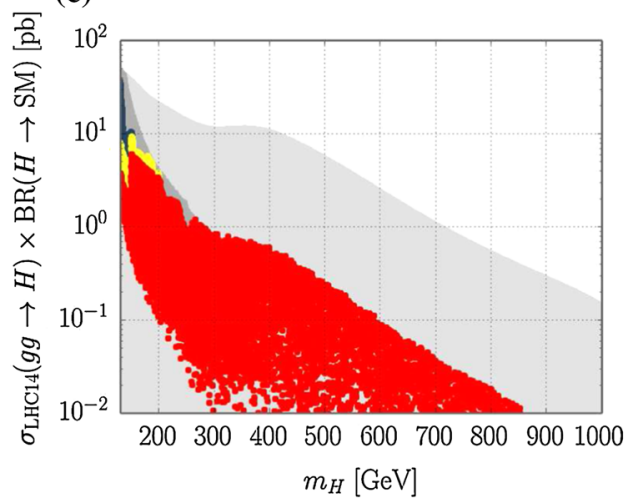

Fig. 13 LHC signal rates of the heavy Higgs boson $H$ decaying into SM particles (a, c) or into two light Higgs bosons, $H \rightarrow h h(\mathbf{b}, \mathbf{d})$, in dependence of the heavy Higgs mass, $m_{H}$, for $\mathrm{CM}$ energies of $8 \mathrm{TeV}$ $(\mathbf{a}, \mathbf{b})$ and $14 \mathrm{TeV}(\mathbf{c}, \mathbf{d})$. a Heavy Higgs signal rate with SM particles in the final state for the LHC at $8 \mathrm{TeV}$. The orange solid (dashed) curves indicate the observed (expected) $95 \%$ CL limits from the latest CMS combination of SM Higgs searches [85]. b Heavy Higgs signal rate with

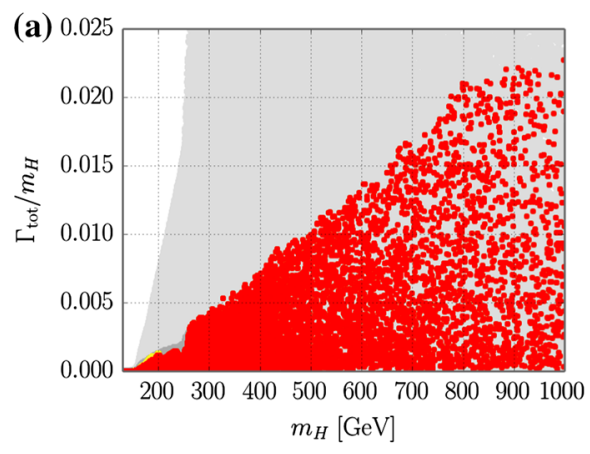

Fig. 14 Total width, $\Gamma_{\text {tot }}$, as a function of the Higgs mass $m_{H}$. We display the ratio, $\Gamma_{\text {tot }} / m_{H}(\mathbf{a})$, as well as the suppression factor with respect to the SM Higgs width, $\Gamma_{\text {tot }} / \Gamma_{\text {tot, SM }}(\mathbf{b})$. We obtain $\Gamma_{\text {tot }} / m_{H} \lesssim 0.02$, as well as a suppression of $25 \%$ or lower of the total width compared to

as well as from direct searches for the $H \rightarrow h h$ process with $\gamma \gamma b \bar{b}$ [99] and $b \bar{b} b \bar{b}$ [100] final states. We see that at the current stage, the experimental searches with SM-like final states yield important constraints for $m_{H} \lesssim 300 \mathrm{GeV}$. As

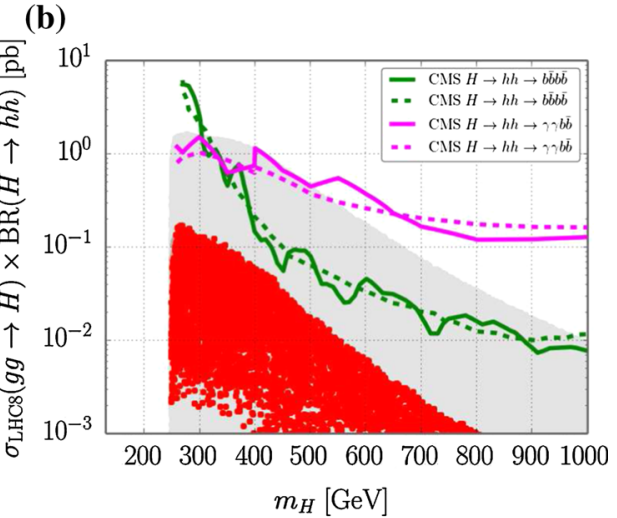

(d)

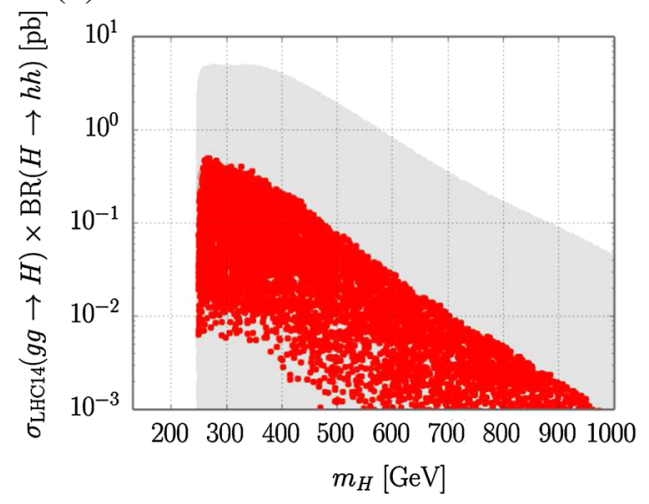

light Higgs bosons in the final state for the LHC at $8 \mathrm{TeV}$. We display the current expected and observed $95 \%$ CL limits from CMS $H \rightarrow h h$ searches with $\gamma \gamma b \bar{b}$ [99] and $b \bar{b} b \bar{b}$ [100]. c Heavy Higgs signal rate with SM particles in the final state for the LHC at $14 \mathrm{TeV}$. d Heavy Higgs signal rate with light Higgs bosons in the final state for the LHC at $14 \mathrm{TeV}$

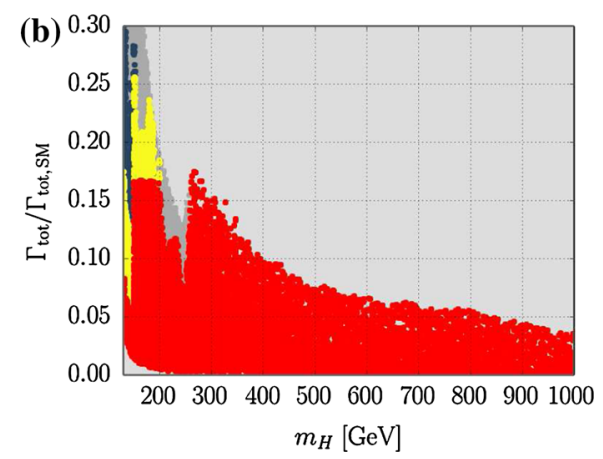

the SM prediction, in agreement with Ref. [41]. a Ratio of total width, $\Gamma_{\text {tot }}$, and the Higgs mass, $m_{H}$. b Suppression of the total width with respect the total width of a SM Higgs boson at mass $m_{H}$

discussed above, at larger masses the upper limit on the mixing angle, and thus on the maximal production cross section, stems either from $m_{W}$ or from perturbativity. Note that the displayed CMS limit from the SM Higgs search combina- 
(a) allowed scale factor and total width, $\mu=4 \times 10^{10} \mathrm{GeV}$
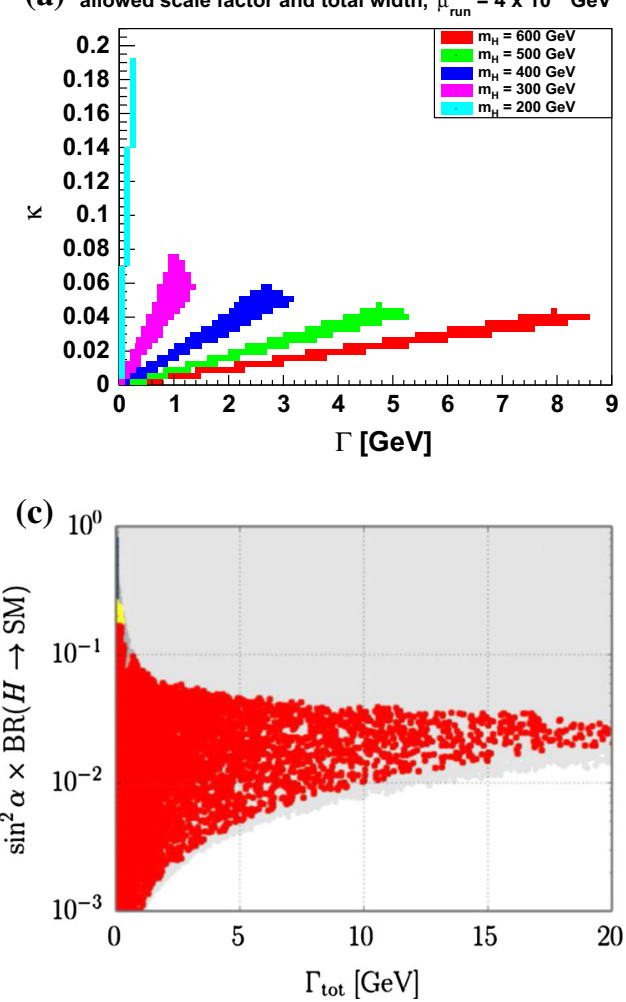

Fig. 15 Allowed regions in the $\left(\Gamma_{\text {tot }}, \kappa\right)$ plane $(\mathbf{a}, \mathbf{c})$ and $\left(\Gamma_{\text {tot }}, \kappa^{\prime}\right)$ plane $(\mathbf{b}, \mathbf{d})$. In $\mathbf{a}, \mathbf{b}$ the results are shown for various fixed values of $m_{H} \cdot \kappa$ and $\kappa^{\prime}$ denote the scaling factors for SM-like decays and the new physics channel $H \rightarrow h h$, respectively, cf. Eqs. (32) and (33). a ( $\left.\Gamma_{\text {tot, }, k}\right)$ plane

tion [85] is only based on $\leq 5.1 \mathrm{fb}^{-1}$ of $7 \mathrm{TeV}$ and $\leq 12.2 \mathrm{fb}^{-1}$ of $8 \mathrm{TeV}$ data, thus not exploiting the full available data from LHC run 1. Obviously, future LHC searches for a SM-like Higgs boson with reduced couplings in the full accessible mass range will play an important role in probing the singletextended SM. The direct searches for the $H \rightarrow h h$ process carried out by CMS in the final states $\gamma \gamma b \bar{b}$ [99] and, in particular, $b \bar{b} b \bar{b}[100]$ draw near to the allowed region at masses $\sim 450 \mathrm{GeV}$. While they do not yield any relevant constraints at the current stage, these searches will become important in this model in the upcoming LHC runs, as they are complementary to the SM-like Higgs searches. For reference, we also provide the predicted LHC signal cross section for both the SM Higgs signatures and $H \rightarrow h h$ signature for $\mathrm{CM}$ energies of 8 and $14 \mathrm{TeV}$ in Fig. 13. Note that, as discussed earlier (see footnote 7), we do not include effects from the interference with the Higgs boson at $\sim 125 \mathrm{GeV}$ in these predictions.

In general, the total width of the heavy Higgs boson is of high interest for collider searches. In the SM, the width of the SM Higgs boson rapidly rises with its mass. In Ref. [41] it was shown that in the singlet-extended SM the total width of the heavy resonance, $\Gamma_{\text {tot }}\left(m_{H}\right)$, is highly suppressed due
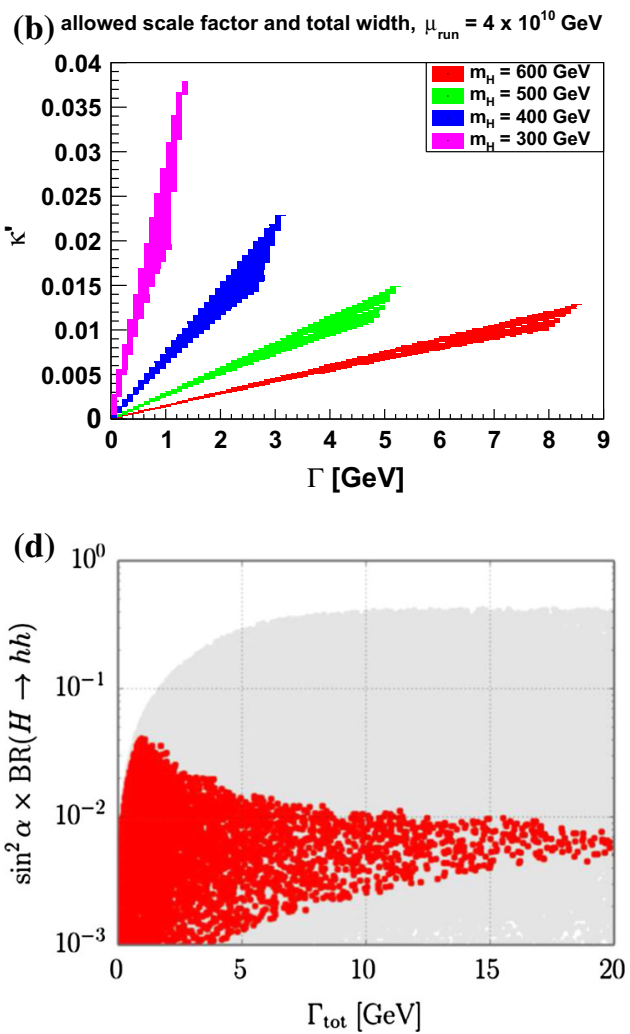

for fixed Higgs mass values $m_{H} \cdot \mathbf{b}\left(\Gamma_{\text {tot }, k^{\prime}}\right)$ plane for fixed Higgs mass values $m_{H}$. c $\left(\Gamma_{\text {tot }, k}\right)$ plane from the full scan. $\mathbf{d}\left(\Gamma_{\text {tot }, k^{\prime}}\right)$ plane from the full scan

to the small mixing angle required. The same behavior is observed here. We show the ratio $\Gamma_{\text {tot }} / m_{H}$, as well as the suppression of the width, $\Gamma_{\text {tot }} / \Gamma_{\mathrm{SM}}$, in Fig. 14 . We see that the total width of the heavy Higgs only amounts to up to $\sim 20-25 \%$ at lower masses $m_{H} \lesssim 200 \mathrm{GeV}$, while it is even further suppressed to below 5-15\% of the SM Higgs width for masses $m_{H}>300 \mathrm{GeV}$. At $m_{H}=1000 \mathrm{GeV}$, the total width is still below $25 \mathrm{GeV}$. In comparison to SM Higgs boson of the same mass, the total width of these resonances is therefore highly suppressed, which promises to enhance the validity of a narrow width approximation in this mass range. ${ }^{13}$

For completeness, we show the allowed parameter space in the $\left(\Gamma_{\text {tot }}, \kappa\right)$ and $\left(\Gamma_{\text {tot }}, \kappa^{\prime}\right)$ planes in Fig. 15 . If these predictions are taken as independent input parameters in future Higgs boson collider searches, a direct comparison with the experimental results renders additional constraints and-in case of a discovery - could possibly lead to an exclusion of the entire model.

\footnotetext{
13 See e.g. Ref. [75] for the discussion of finite width effects for SM-like Higgs bosons in the mass range $m_{h} \gtrsim 200 \mathrm{GeV}$.
} 


\subsection{Low mass region}

We now consider the low mass region, i.e. we set the heavy Higgs mass to $m_{H}=125.14 \mathrm{GeV}$ and investigate the parameter space with $m_{h} \in[1,120] \mathrm{GeV}$. In contrast to the high mass region, results from LEP searches play an important role in this part of parameter space. As discussed in Sect. 3.3, we here do not apply limits from RGE running of the couplings. Before constraints from the signal rates are taken into account, this a priori leads to much larger allowed values for $\tan \beta$, where the upper limit on $\tan \beta$ stems from perturbative unitarity, cf. Fig. 1. However, whenever the additional decay $H \rightarrow h h$ is kinematically allowed, $\tan \beta$ values $\gtrsim 1$ generally result in large branching ratios for this channel, cf. Fig. 5a. This immediately imposes a quite strong suppression of the SM decays of the heavy Higgs state, leading to strong bounds on the minimal $|\sin \alpha|$ value from the signal rates, cf. Sect. 3.6. However, we should keep in mind that in parameter regions where $\tan \beta \approx-\cos \alpha / \sin \alpha$, the branching ratio for $H \rightarrow h h$ decreases significantly, thus restoring the signal strength of the heavy Higgs boson to $\sin ^{2} \alpha$ times the SM Higgs signal strength. In the mass range where the additional decay is not allowed and up to values of $m_{h} \lesssim 100 \mathrm{GeV}$, the strongest limits on the mixing angle stem from LEP Higgs searches in the channel $e^{+} e^{-} \rightarrow Z h \rightarrow Z(b \bar{b})$ [92]. For larger Higgs masses, the Higgs signal rates yield stricter limits on $|\sin \alpha|$ than the exclusion limits from LEP and LHC, cf. also Fig. 7. We have summarized our finding in the low mass scenario in Table 3.

We now turn to the discussion of the full scan. In order to highlight the importance of LEP constraints in the low mass region, we employ the following color coding for the plots:

- Light gray points which fail theoretical constraints.

- Dark gray points which are excluded by LHC Higgs searches.

- Blue points allowed by LHC Higgs searches, but excluded by $>95 \%$ CL by LEP searches.

- Dark green points consistent with LEP constraints within $2 \sigma$.

- Light green points consistent with LEP constraints within $1 \sigma$.

- Yellow points favored within $2 \sigma$ in the global fit (HiggsSignals $\chi^{2}+\operatorname{LEP} \chi^{2}$ ).

- red points favored within $1 \sigma$ in the global fit (HiggsSignals $\chi^{2}+\operatorname{LEP} \chi^{2}$ ).

The results are shown in Fig. 16 in terms of twodimensional scatter plots in the scan parameters. In Fig. 16c, we see that most parameter points allowed by the global fit at the $2 \sigma$ level are found for $m_{h} \gtrsim 80 \mathrm{GeV}$ and $|\sin \alpha| \gtrsim 0.85$. For lower Higgs masses the mixing angle is constrained to values very close to the decoupling scenario $(|\sin \alpha| \approx 1)$.
Table 3 Limits on $\sin \alpha$ and $\tan \beta$ in the low mass scenario for various light Higgs masses $m_{h}$. The limits on $\sin \alpha$ have been determined at $\tan \beta=1$. The lower limit on $\sin \alpha$ stemming from exclusion limits from LEP or LHC Higgs searches evaluated with HiggsBounds is given in the second column. If the lower limit on $\sin \alpha$ obtained from the test against the Higgs signal rates using HiggsSignals results in stricter limits, we display them in the third column. The upper limit on $\tan \beta$ in the fourth column stems from perturbative unitarity for the complete decoupling case $(|\sin \alpha|=1)$, cf. Fig. 1 . In the fifth column we give the $\tan \beta$ value for which $\Gamma_{H \rightarrow h h}=0$ is obtained, given the maximal mixing angle allowed by the Higgs exclusion limits (second column). At this $\tan \beta$ value, the $|\sin \alpha|$ limit obtained from the Higgs signal rates (third column) is abrogated

\begin{tabular}{lllcl}
\hline$m_{h}(\mathrm{GeV})$ & $|\sin \alpha|_{\min , \mathrm{HB}}$ & $|\sin \alpha|_{\min , \mathrm{HS}}$ & $(\tan \beta)_{\max }$ & $(\tan \beta)_{\mathrm{no} H \rightarrow h h}$ \\
\hline 120 & 0.410 & 0.918 & 8.4 & - \\
110 & 0.819 & 0.932 & 9.3 & - \\
100 & 0.852 & 0.891 & 10.1 & - \\
90 & 0.901 & - & 11.2 & - \\
80 & 0.974 & - & 12.6 & - \\
70 & 0.985 & - & 14.4 & - \\
60 & 0.978 & 0.996 & 16.8 & 0.21 \\
50 & 0.981 & 0.998 & 20.2 & 0.20 \\
40 & 0.984 & 0.998 & 25.2 & 0.18 \\
30 & 0.988 & 0.998 & 33.6 & 0.16 \\
20 & 0.993 & 0.998 & 50.4 & 0.12 \\
10 & 0.997 & 0.998 & 100.8 & 0.08 \\
\hline
\end{tabular}

The LEP limits are particularly strong in the mass region between 65 and $72 \mathrm{GeV}$, cf. Fig. 4a, such that only a few valid points are found here, as can be seen best in Fig. 16b. The semi-oval exclusion region in Fig. 16a, $\mathrm{c}$ for large $\tan \beta$ values and low $|\sin \alpha|$ values, respectively, corresponds to a $2 \sigma$ deviation in the electroweak oblique parameters $S, T$, and $U$.

In Fig. 16b, we observe a drastic change in the distribution of allowed parameter points when going to Higgs masses $m_{h}<m_{H} / 2 \approx 62 \mathrm{GeV}$, where the decay mode $H \rightarrow h h$ becomes kinematically accessible. As discussed earlier in Sect. 3.6, cf. Fig. 5, the decay $H \rightarrow h$ h easily becomes the dominant decay mode if $\tan \beta \gtrsim 1$, unless the mixing angle is very close to $|\sin \alpha|=1$. Hence, for $m_{h}<m_{H} / 2$, most of the allowed points are found for small values of $\tan \beta$, since the Higgs signal rates favor small values of $\mathrm{BR}(H \rightarrow h h)$. At larger Higgs masses, $m_{h}>m_{H} / 2$, the favored points are equally distributed over the entire $\tan \beta$ range allowed by perturbative unitarity.

It is interesting to investigate the allowed range of the $H \rightarrow h h$ signal rate in dependence of the light Higgs mass. This is shown in Fig. 17a, where the signal rate is normalized to the SM Higgs boson production. Note that, due to the LEP constraints, the favored points feature a mixing angle $|\sin \alpha| \approx 1$ and thus the displayed signal rate closely resembles $\operatorname{BR}(H \rightarrow h h)$. We see that the maximally allowed 

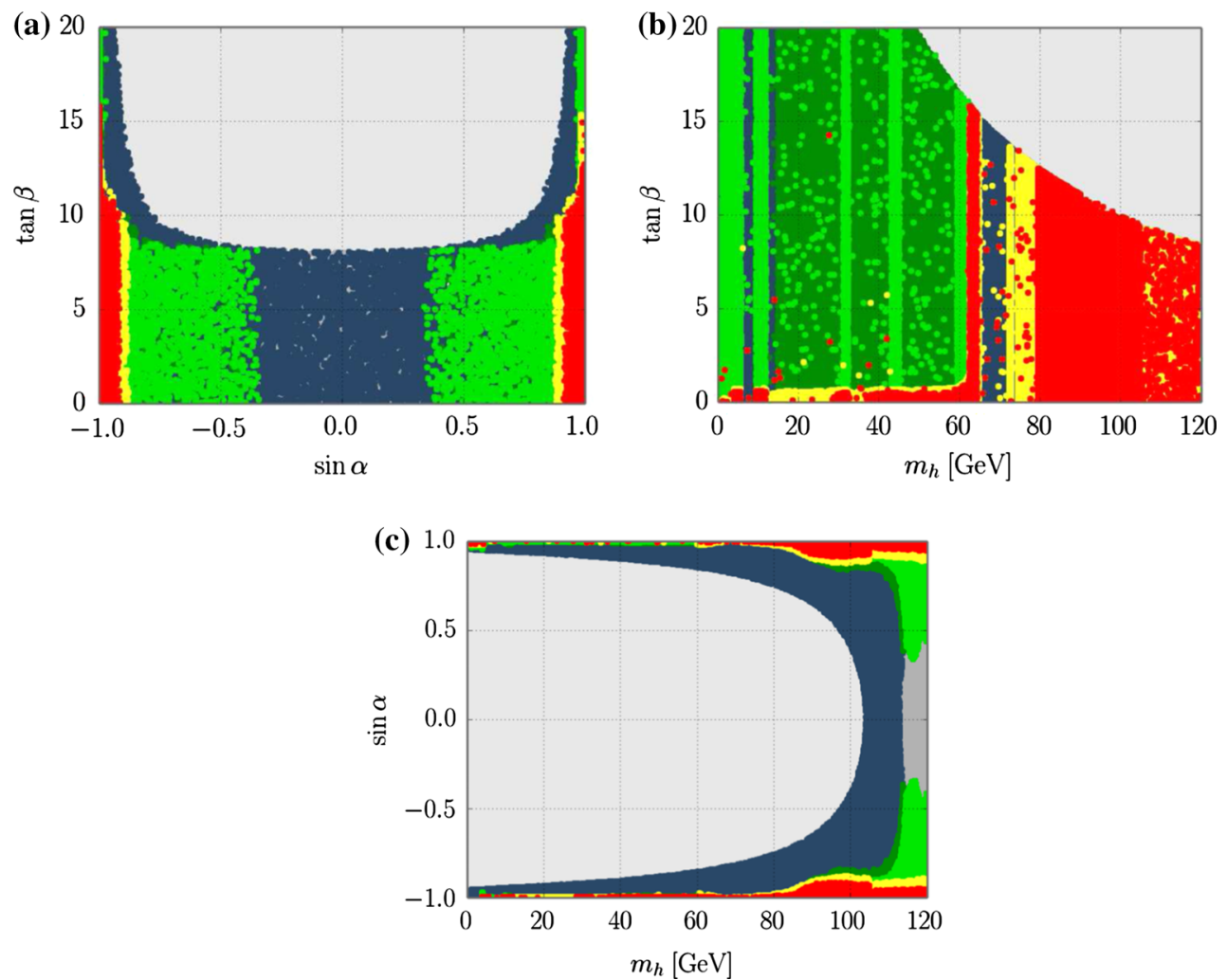

Fig. 16 Two-dimensional parameter correlations between $m_{H}, \tan \beta$, and $\sin \alpha$ in the low mass region. See text for a description of the color coding. a $(\sin \alpha, \tan \beta)$ plane. b $\left(m_{h}, \tan \beta\right)$ plane. c $\left(m_{h}, \sin \alpha\right)$ plane

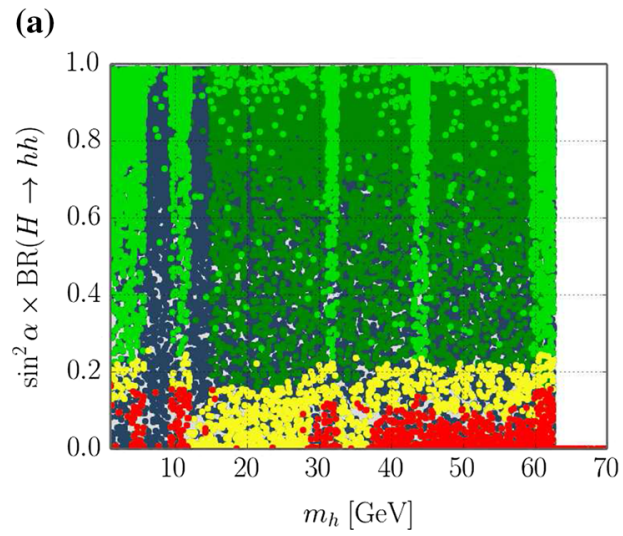

Fig. 17 Signal rates for the $H \rightarrow h h$ signature in dependence of the light Higgs mass: a Signal rate for the $H \rightarrow h h$ signature, normalized to the SM Higgs production cross section. b Signal rate for the

$H \rightarrow h$ signal rate is about $22 \%$ and is roughly independent on the light Higgs mass. ${ }^{14}$ This upper limit solely stems from the observed signal rates of the SM-like Higgs

\footnotetext{
$\overline{14}$ The reason why the density of allowed points still depends strongly on $m_{h}$ is that regions which are strongly constrained by LEP searches require a large fine-tuning of $\sin \alpha$ to render allowed points.
}

(b)

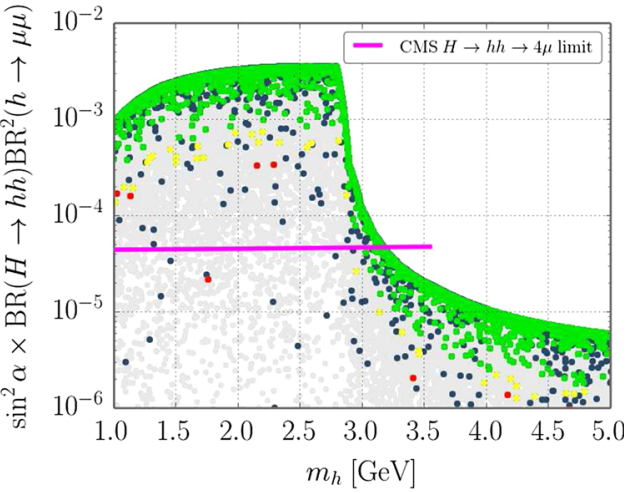

$H \rightarrow h h \rightarrow \mu^{+} \mu^{-} \mu^{+} \mu^{-}$signature at very low masses, normalized to the SM Higgs production cross section. The magenta line indicates the observed limit from a CMS $8 \mathrm{TeV}$ analysis [94]

boson at $\sim 125 \mathrm{GeV}$. These constraints therefore also limit the total width of the heavy Higgs at $125.14 \mathrm{GeV}$ to values $\sim 3-5 \mathrm{MeV}$, being in the vicinity of the SM total width of $\sim 4.1 \mathrm{MeV}$.

We now discuss the case of very low Higgs masses, $m_{h} \lesssim$ $4 \mathrm{GeV}$. Here, the LEP constraints stem from the decay-mode independent analysis of $e^{+} e^{-} \rightarrow$ Zh by OPAL [93], yielding 
Table 4 Constraints from $B$-factories on a light Higgs boson with mass $m_{h}$. The second to fifth column list the current experimental $90 \% \mathrm{CL}$ upper bounds on the decay rate of $\Upsilon(1 s) \rightarrow h \gamma$ and successive Higgs decay (specified in the second title row). The inferred upper limit on the rescaling factor of the bottom Yukawa coupling in given in the sixth col- umn, and-if possible- the lower limit on the singlet-doublet mixing angle $|\sin \alpha|$ is given in the last column. We indicate the most relevant constraint for the model (yielding the listed limits on the model parameters) by bold numbers

\begin{tabular}{|c|c|c|c|c|c|c|}
\hline \multirow[t]{2}{*}{$m_{h}[\mathrm{GeV}]$} & \multicolumn{4}{|c|}{$90 \%$ CL upper limit on $\mathrm{BR}(\Upsilon(1 s) \rightarrow h \gamma, h \rightarrow \cdots)$} & \multirow[t]{2}{*}{$g_{b}^{2}$ (upper limit) } & \multirow[t]{2}{*}{$|\sin \alpha|$ (lower limit) } \\
\hline & $h \rightarrow \mu^{+} \mu^{-}[111]$ & $h \rightarrow \tau^{+} \tau^{-}[109]$ & $h \rightarrow g g[112]$ & $h \rightarrow s \bar{s}[112]$ & & \\
\hline 1.0 & $\sim 4 \times 10^{-6}$ & - & $\sim 5 \times 10^{-6}$ & - & $\sim 0.25$ & $\sim 0.87$ \\
\hline 2.0 & $\sim 5 \times 10^{-6}$ & - & $\sim 1 \times 10^{-4}$ & $\sim 5 \times 10^{-5}$ & $\sim 1.16$ & - \\
\hline 3.0 & $\sim 6 \times 10^{-6}$ & - & $\sim 2 \times 10^{-4}$ & $\sim 8 \times 10^{-5}$ & $\sim 7.82$ & - \\
\hline 4.0 & $\sim 8 \times 10^{-6}$ & $1.2 \times 10^{-5}$ & $\sim 4 \times 10^{-4}$ & $\sim 3 \times 10^{-4}$ & 2.06 & - \\
\hline 5.0 & $\sim 8 \times 10^{-6}$ & $9.1 \times 10^{-6}$ & $\sim 3 \times 10^{-4}$ & $\sim 3 \times 10^{-4}$ & 0.68 & 0.57 \\
\hline 6.0 & $\sim 1 \times 10^{-5}$ & $2.3 \times 10^{-5}$ & $\sim 5 \times 10^{-5}$ & $\sim 8 \times 10^{-5}$ & 1.59 & - \\
\hline 7.0 & $\sim 1 \times 10^{-5}$ & $1.6 \times 10^{-5}$ & $\sim 3 \times 10^{-4}$ & $\sim 1 \times 10^{-4}$ & 1.33 & - \\
\hline 8.0 & $\sim 2 \times 10^{-5}$ & $3.2 \times 10^{-5}$ & $\sim 1 \times 10^{-2}$ & $\sim 4 \times 10^{-5}$ & 4.45 & - \\
\hline
\end{tabular}

a slightly weaker limit on the mixing angle, $|\sin \alpha| \gtrsim 0.965$, than at larger masses, cf. Fig. 4a. In the mass region $m_{h} \in$ $[1,3] \mathrm{GeV}$, the branching fraction for the light Higgs decay $h \rightarrow \mu \mu$ amounts between 3-6\%, thus allowing to search for the signature $(p p) \rightarrow H \rightarrow h h \rightarrow \mu^{+} \mu^{-} \mu^{+} \mu^{-}$at the LHC. We show the predicted signal rate for this signature ${ }^{15}$ for the LHC at a center-of-mass energy of $8 \mathrm{TeV}$ in Fig. 17b. A search for this signature has been performed by CMS [94], yielding the observed upper limit $^{16}$ displayed as magenta line in the figure. As can be seen, the CMS limit provides competitive constraints in this parameter region, excluding a sizable amount of the parameter region favored by the global fit. Future LHC searches for the $4 \mu$ signature therefore have a good discovery potential in this mass region. Other final states, composed of $\tau$ leptons, strange or charm quarks, could be exploited at a future linear $e^{+} e^{-}$collider like the ILC.

A very light Higgs boson $h$ with mass values up to the $b \bar{b}$ threshold can also be probed at $B$-factories in the radiative decay $\Upsilon \rightarrow h \gamma$ [103], with successive decay of the light Higgs boson to $\tau$-lepton, muon or hadron pairs. Here, we provide a rough estimate of the present constraints.

The decay rate for the $1^{--}$bound state $\Upsilon(1 s)$ to the Higgs-photon final state (normalized to the decay rate of $\left.\Upsilon(1 s) \rightarrow \mu^{+} \mu^{-}\right)$is given by [103]

$$
\frac{\operatorname{BR}(\Upsilon(1 s) \rightarrow h \gamma)}{\operatorname{BR}\left(\Upsilon(1 s) \rightarrow \mu^{+} \mu^{-}\right)}=\frac{g_{b}^{2} G_{F} m_{b}^{2}}{\sqrt{2} \pi \alpha}\left(1-\frac{m_{h}^{2}}{M_{\Upsilon(1 s)}^{2}}\right) \times \mathcal{F},
$$

\footnotetext{
$\overline{15}$ We consider here only the Higgs production via gluon-gluon fusion.

16 This exclusion limit is not provided with HiggsBounds -4.2 .0 , because the expected limit from the CMS analysis is not publicly available.
}

where $G_{F}$ is the Fermi constant, $m_{b}$ the bottom quark mass, $\alpha$ the fine-structure constant and $\operatorname{BR}\left(\Upsilon(1 s) \rightarrow \mu^{+} \mu^{-}\right) \approx$ $2.48 \pm 0.05 \%$ [104]. The factor $\mathcal{F}$ represents higher-order corrections. The one-loop QCD corrections have been calculated in Refs. [105,106] and are known to reduce the leadingorder estimate by up to $84 \%$; see Ref. [107] for an extended discussion. In our model, the rescaling factor of the bottom Yukawa coupling of the light Higgs is simply given by $g_{b}=\cos \alpha$.

Recent experimental searches have been carried out by BaBar [108-112] and CLEO [113], focusing on the search for a light $\mathcal{C} \mathcal{P}$-odd Higgs boson motivated by certain nextto-minimal supersymmetric standard model scenarios [114116]. The $90 \%$ CL upper limits on the branching fraction of these search signatures are typically of $\sim \mathcal{O}\left(10^{-4}-10^{-6}\right)$ and are listed for representative values of the light Higgs mass in Table 4 (cf. also Refs. [117-119] for more details). Generally, these limits underlie large statistical fluctuations, thus we prefer to use a roughly estimated mean value and indicate this by a ' $\sim$ ' in front of the quoted number. Using the SM Higgs boson branching ratios for $h \rightarrow \mu^{+} \mu^{-}, h \rightarrow \tau^{+} \tau^{-}$, $h \rightarrow g g$, and $h \rightarrow s \bar{s}$ in this mass region, we can infer a $90 \%$ C.L upper limit on the rescaling factor of the bottom Yukawa coupling, $g_{b}$, which is listed in Table 4. If this upper limit is below 1, we furthermore quote the resulting lower limit on the mixing angle $|\sin \alpha|$ in the table. The resulting limits cannot compete with those obtained from direct LEP searches, however, future $B$-physics facilities such as the Belle II experiment at the Super KEKB accelerator [120] will be able to probe the yet unexcluded region.

Finally, we want to comment that despite of the quite strong constraints in the low mass region, a substantial number of low mass Higgs bosons could already have been directly produced at the LHC. Table 5 exemplarily lists the 
Table 5 Maximally allowed cross sections, $\sigma_{g g}=\left(\cos ^{2} \alpha\right)_{\max } \times$ $\sigma_{g g, \mathrm{SM}}$, for direct light Higgs production at the LHC at CM energies of 8 and $14 \mathrm{TeV}$ after all current constraints have been taken into account. The SM Higgs production cross sections have been taken from Refs. [35,121]

\begin{tabular}{llc}
\hline$m_{h}(\mathrm{GeV})$ & $\sigma_{g g}^{8 \mathrm{TeV}}(\mathrm{pb})$ & $\sigma_{g g}^{14 \mathrm{TeV}}(\mathrm{pb})$ \\
\hline 120 & 3.28 & 8.40 \\
110 & 3.24 & 8.12 \\
100 & 6.12 & 14.96 \\
90 & 6.82 & 16.26 \\
80 & 2.33 & 5.41 \\
70 & 2.97 & 6.73 \\
60 & 0.63 & 1.38 \\
50 & 0.45 & 0.96 \\
40 & 0.74 & 1.50
\end{tabular}

maximally allowed LHC cross sections for direct production in gluon-gluon fusion for a selected range of light Higgs masses at $\mathrm{CM}$ energies of 8 and $14 \mathrm{TeV} .{ }^{17}$ We encourage the LHC experiments to explore the feasibility of experimental searches within the low mass region and to potentially extend the searches for directly produced scalars into this mass range.

\subsection{Intermediate mass region}

For the intermediate mass region, which contains the special case of mass-degenerate Higgs states, we treat both Higgs masses as free parameters in the fit, $m_{h}, m_{H} \in$ $[120,130] \mathrm{GeV}$. Note that the following discussion is based on a few simplifying assumptions about overlapping Higgs signals in the experimental analyses. It should be clear that a precise investigation of the near mass-degenerate Higgs scenario can only be performed by analyzing the LHC data directly and is thus restricted to be done by the experimental collaborations (see e.g. Ref. [7] for such an analysis). Nevertheless, we want to point out this interesting possibility here and encourage the LHC experiments for further investigations.

If the Higgs states have very similar masses, their signals cannot be clearly distinguished in the experimental analyses and (to first approximation) the sum of the signal rates has to be considered for the comparison with the measured rates. Moreover, the observed peak in the invariant mass distribution in the $H \rightarrow \gamma \gamma$ and $H \rightarrow Z Z^{*} \rightarrow 4 \ell$ channels, which is fitted to determine the Higgs mass, would actually comprise two (partially) overlapping Higgs resonances, where the height of each resonance is governed by the corresponding

\footnotetext{
17 We thank M. Grazzini for providing us with the production cross sections for $m_{h}<80 \mathrm{GeV}$.
}

signal strength. Therefore, for each Higgs analysis where a mass measurement has been performed, cf. Table 1, we calculate a signal strength weighted mean value of the Higgs masses, ${ }^{18}$

$\bar{m}=\frac{\mu_{h} \cdot m_{h}+\mu_{H} \cdot m_{H}}{\mu_{h}+\mu_{H}}$,

to be tested against the measurement, where the SM normalized signal strengths are given by

$\mu_{h / H}=\frac{\sum_{a} \epsilon^{a} \sigma_{a}\left(m_{h / H}\right) \times \mathrm{BR}_{a}\left(m_{h / H}\right)}{\sum_{a} \epsilon^{a} \sigma_{\mathrm{SM}}^{a}(\hat{m}) \times \mathrm{BR}_{\mathrm{SM}}^{a}(\hat{m})}$.

Here, $\hat{m}$ denotes the mass value hypothesized by the experiment during to signal rate measurement. The index $a$ runs over all signal channels, i.e. Higgs production times decay mode, considered in the experimental analysis, and $\epsilon^{a}$ denotes the corresponding efficiencies. The predicted cross sections $\sigma$ and partial widths are obtained from rescaling the respective SM quantities $[35,101,102]$ by $\cos ^{2} \alpha$ and $\sin ^{2} \alpha$ for $h$ and $H$, respectively. As mentioned earlier in Sect. 3.6, the SM normalized signal strengths $\mu_{h / H}$ contain a slight mass dependence ${ }^{19}$ since the SM cross sections and branching ratios are not constant over the relevant mass range.

We present limits on $\sin \alpha$ and $\tan \beta$ for various choices of the Higgs mass $m$ in the intermediate mass region in Table 6. We fixed the other Higgs mass to $125.14 \mathrm{GeV}$. Depending on whether or not the Higgs mass $m$ is larger than $125.14 \mathrm{GeV}$, we obtain either an upper or lower limit on $\sin \alpha$ from the LHC Higgs search exclusion limits or signal rate measurements, which are listed separately. In the case of nearly degenerate Higgs masses, $m=125 \mathrm{GeV}$, no limit on $\sin \alpha$ can be obtained, since the Higgs signals completely overlap. We find that no limits from $95 \%$ CL exclusions from Higgs searches can be obtained for Higgs masses within 121 and $127 \mathrm{GeV}$. Moreover, the limits inferred from the signal rates become weaker the closer $m$ is to $125.14 \mathrm{GeV}$ due to the signal overlap. In the full intermediate mass region, the limits inferred from the Higgs signal rates supersede the limits obtained from null results in LHC Higgs searches.

The upper limits on $\tan \beta$ listed in Table 6 correspond to the perturbative unitarity bound (cf. Fig. 1). Similarly as in

\footnotetext{
18 Testing overlapping signals of multiple Higgs bosons against mass measurements by employing a mass average calculation is the default procedure in Higgs Signals since version 1.3.0.

19 This mass dependence is neglected per default in Higgs Signals since additional complications arise if theoretical mass uncertainties are present. This is, however, not the case here, since we use the Higgs masses directly as input parameters. The evaluation of the signal strength according to Eq. (36) can be activated in HiggsSignals by setting normalize_rates_to_ reference_position=.True. in the file usefulbits_ HS. $f 90$.
} 
Table 6 Limits on $\sin \alpha$ and $\tan \beta$ in the intermediate mass scenario. We fix one Higgs mass at $125.14 \mathrm{GeV}$ and vary the mass of the other Higgs state, $m$. The limit on $\sin \alpha$ that stems from LHC Higgs searches evaluated with HiggsBounds is given in the second column (if available). The limit on $\sin \alpha$ obtained from the test against the Higgs signal rates with HiggsSignals is given in the third column. Note that, depending on the mass hierarchy, we have either an upper or lower limit on $\sin \alpha$, indicated by the " $<$ " and " >", respectively. The upper limit on $\tan \beta$ is given in the fourth column and always stems from perturbative unitarity; see also Fig. 1. Note that we do not impose constraints from perturbativity and vacuum stability at a high energy scale via RGE evolution of the couplings here

\begin{tabular}{llll}
\hline$m(\mathrm{GeV})$ & $|\sin \alpha|_{\mathrm{HB}}$ & $|\sin \alpha|_{\mathrm{HS}}$ & $(\tan \beta)_{\max }$ \\
\hline 130 & $<0.806$ & $<0.370$ & 7.76 \\
129 & $<0.881$ & $<0.373$ & 7.81 \\
128 & $<0.988$ & $<0.377$ & 7.88 \\
127 & - & $<0.381$ & 7.94 \\
126 & - & $<0.552$ & 8.00 \\
125 & - & - & 8.07 \\
124 & - & $>0.793$ & 8.13 \\
123 & - & $>0.864$ & 8.20 \\
122 & - & $>0.904$ & 8.26 \\
121 & - & $>0.913$ & 8.34 \\
120 & $>0.410$ & $>0.918$ & 8.41 \\
\hline
\end{tabular}

the low mass region, we do not impose constraints from perturbativity and vacuum stability at a high energy scale here. If these were additionally required, $\tan \beta$ would be limited to values $\lesssim 1.86$ for $m \geq 125.14 \mathrm{GeV}$. For lower Higgs masses $m$ no valid points would be found. It should be noted, however, that the collider phenomenology does not depend on $\tan \beta$ in the intermediate mass region, since Higgs-to-Higgs decays are kinematically not accessible.

The results from the full four-dimensional scan are presented in Fig. 18 in terms of two-dimensional scatter plots, using the same color coding as in the high mass region (see e.g. Fig. 10). The correlation between the two Higgs masses, Fig. 18a, shows that allowed parameter points with Higgs bosons in the full intermediate mass region are found, however, at least one of the Higgs masses is always required to be roughly between 124 and $126.5 \mathrm{GeV}$. We can furthermore learn from Fig. 18c, d that allowed points with one Higgs mass being below $124 \mathrm{GeV}$ (above $126.5 \mathrm{GeV}$ ) feature $|\sin \alpha|$ values close to $1(0)$, such that the other Higgs state at around $125 \mathrm{GeV}$ has SM Higgs-like signal strengths. In the near-degenerate case, $m_{h} \approx m_{H} \approx 125 \mathrm{GeV}$, all mixing angles $\sin \alpha$ are allowed and the model appears indistinguishable from the SM at current collider experiments.

Figure 18 also shows the correlations of $\tan \beta$ with the mixing angle $\sin \alpha$, Fig. 18b, and the Higgs masses, Fig. 18e, $\mathrm{f}$. As stated earlier, $\tan \beta$ does not influence the collider phenomenology in the intermediate mass range, thus we find allowed parameter points in the full $\tan \beta$ range up to the maximal value given by perturbative unitarity.

A direct search for the second Higgs boson in the intermediate mass region at the LHC seems challenging. Even if the mass splitting between the two Higgs states is large enough to be resolved by the experimental analyses, we expect the second resonance to be much smaller than the established signal. Nevertheless we would like to encourage the LHC experiments to perform dedicated resonance searches, in particular in the mass region slightly above the current signal, $m_{H} \sim(125.5-126.5) \mathrm{GeV}$, since in this case larger values of the mixing angle are still allowed while an improvement of the vacuum stability at the high scale may be obtained. More promising prospects to resolve the near mass-degenerate Higgs scenario have future experimental facilities like the ILC $[18,42]$ or a muon collider $[42,98]$, where the latter provides excellent opportunities to measure the mass and the total width of the discovered Higgs boson via a line-shape scan.

\section{Conclusions}

In this work, we have investigated the theoretical and experimental limits on the parameter space of a real singlet extension of the SM Higgs sector, considering mass values of the second Higgs boson ranging from $1 \mathrm{GeV}$ to $1 \mathrm{TeV}$, i.e. within the accessible mass range of past, current and future collider experiments. This study complements a previous work [41], which was restricted to $m_{H} \in[600 \mathrm{GeV}, 1 \mathrm{TeV}]$ and, moreover, did not include constraints from direct Higgs collider searches. In the present work, either the heavy or the light Higgs state can take the role of the discovered SM-like Higgs boson at $125 \mathrm{GeV}$. We found that up to Higgs masses $m \lesssim 300 \mathrm{GeV}$, exclusion limits from direct Higgs collider searches at LEP and the LHC, as well as the requirement of consistency with the measured SM-like Higgs signal rates pose quite strong constraints. At higher Higgs masses, strong limits stem from electroweak precision observables, in particular from the $W$ boson mass calculated at NLO, as well as from requiring perturbativity of the couplings and vacuum stability. The latter two are tested both at the electroweak scale and at a high scale $\mu \sim 4 \times 10^{10} \mathrm{GeV}$ using the $\beta$ functions of the theory (see e.g. Ref. [41] and references therein).

We performed an exhaustive scan in the three model parameters-specified by the Higgs mixing angle, the second Higgs mass and the ratio of the Higgs VEVs-and provided a detailed discussion of the viable parameter space and the relative importance of the various constraints. We translated these results into predictions for collider observables for the second yet undiscovered Higgs boson, which are 
Fig. 18 Two-dimensional correlations between $m_{h}, m_{H}$, $\sin \alpha$, and $\tan \beta$ in the intermediate mass region. a $\left(m_{h}, m_{H}\right)$ plane. $\mathbf{b}(\sin \alpha, \tan \beta)$ plane. c $\left(m_{h}, \sin \alpha\right)$ plane. d $\left(m_{H}, \sin \alpha\right)$ plane. e $\left(m_{h}, \tan \beta\right)$ plane. $\mathbf{f}\left(m_{H}, \tan \beta\right)$ plane
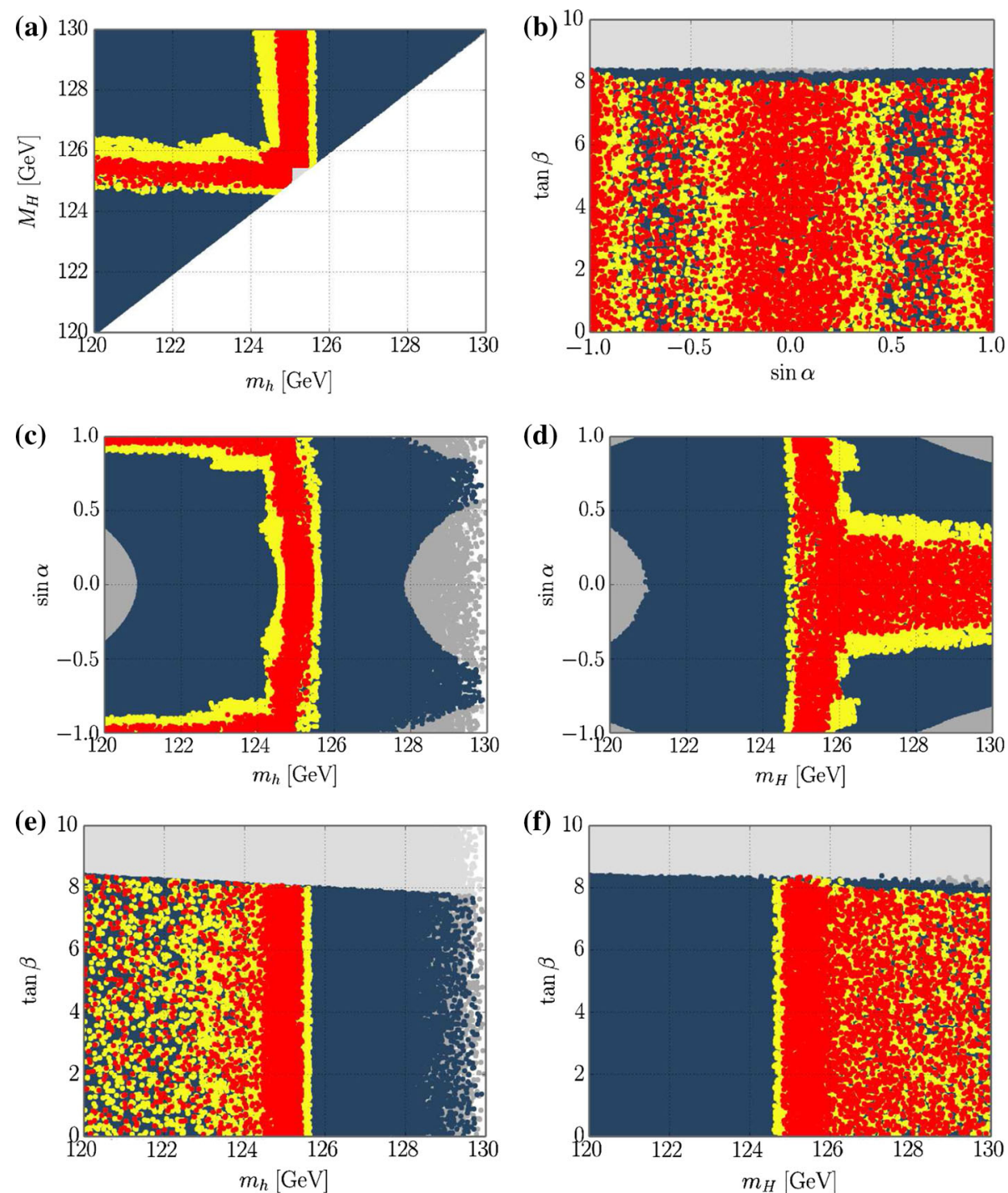

currently investigated by the LHC experiments. In particular, we focused on the global rescaling factor $\kappa$ for the SM Higgs decay modes, the signal rate for the Higgs-to-Higgs decay signature $H \rightarrow h h$ as well as the total width $\Gamma$ of the new scalar. A typical feature of the model is that the total width of the new scalar is quite suppressed with respect to the SM Higgs boson at such masses. At very light Higgs boson masses below $10 \mathrm{GeV}$ we found that new results from LHC searches for the signature $H \rightarrow h h \rightarrow 4 \mu$ are complementary to LEP Higgs searches and thus probe an unexplored parameter region. Also future $B$-factories should be able to probe these parameter regions through the decay $\Upsilon \rightarrow h \gamma$.

We furthermore investigated the intermediate mass region, where both Higgs masses are between 120 and $130 \mathrm{GeV}$, and discussed some of the experimental challenges in probing this scenario. Dedicated LHC searches for an additional resonance in the invariant mass spectra of the $H \rightarrow \gamma \gamma$ (see Ref. [7] for a CMS analysis) and $H \rightarrow Z Z^{*} \rightarrow 4 \ell$ chan- nel in the vicinity of the discovered Higgs boson as well as future precision experiments at the ILC or a muon collider may shed more light onto this case.

The discovery of additional Higgs states is one of the main goals of the upcoming runs of the LHC. In this model, two distinct and complementary signatures of the second Higgs state arise. Firstly, the $H \rightarrow h h$ decay signature, where the best sensitivity for the LHC is obtained for heavy Higgs masses between $250 \mathrm{GeV}$ and roughly $500 \mathrm{GeV}$. These signatures have been recently explored by ATLAS and CMS [99, 100,122] but the analyses are not yet sensitive to constrain the parameter space. Secondly, Higgs searches designed for a SM Higgs boson are sensitive probes of the parameter space. We strongly encourage the experimental collaborations to continue these searches in the full accessible mass range. However, some of the features of the second Higgs state discussed in this work, such as the strong reduction of the total width, should be taken into account in 
upcoming analyses. Finally, we hope that the predictions of LHC signal cross sections at a CM energy of $14 \mathrm{TeV}$ will be found useful for designing some interesting benchmark points for the experimental analyses of this model.

Acknowledgments We thank Klaus Desch and Sven Heinemeyer for inspiring remarks and for motivating us to perform this study. We furthermore acknowledge helpful discussions with Philip Bechtle, Howie Haber, Antonio Morais, Marco Sampaio, Rui Santos and Martin Wiebusch. The code for testing perturbative unitarity has been adapted from Ref. [41]. TS is supported in part by US Department of Energy Grant No. DE-FG02-04ER41286, and in part by a FeodorLynen research fellowship sponsored by the Alexander von Humboldt Foundation.

Open Access This article is distributed under the terms of the Creative Commons Attribution License which permits any use, distribution, and reproduction in any medium, provided the original author(s) and the source are credited.

Funded by $\mathrm{SCOAP}^{3}$ / License Version CC BY 4.0.

\section{Appendix A: Minimization and vacuum stability conditions}

In this appendix we briefly guide the reader through the steps from Eqs. (3) to (4), using the definition of the scalar fields given in Eq. (6). We basically follow the discussion as presented in Ref. [41].

With the definition of the VEVs according to Eq. (6), the extrema of $V$ are determined using the following set of equations:

$$
\begin{aligned}
& \frac{\partial V}{\partial v}(v, x)=v \cdot\left(-m^{2}+\lambda_{1} v^{2}+\frac{\lambda_{3}}{2} x^{2}\right)=0 \\
& \frac{\partial V}{\partial x}(v, x)=x \cdot\left(-\mu^{2}+\lambda_{2} x^{2}+\frac{\lambda_{3}}{2} v^{2}\right)=0 .
\end{aligned}
$$

The physically interesting solutions have $v, x>0$ :

$$
\begin{aligned}
& v^{2}=\frac{\lambda_{2} m^{2}-\frac{\lambda_{3}}{2} \mu^{2}}{\lambda_{1} \lambda_{2}-\frac{\lambda_{3}{ }^{2}}{4}}, \\
& x^{2}=\frac{\lambda_{1} \mu^{2}-\frac{\lambda_{3}}{2} m^{2}}{\lambda_{1} \lambda_{2}-\frac{\lambda_{3}{ }^{2}}{4}} .
\end{aligned}
$$

Alternatively, we use Eq. (37) to eliminate $m^{2}$ and $\mu^{2}$, leading to

$m^{2}=\lambda_{1} v^{2}+\frac{\lambda_{3}}{2} x^{2}, \quad \mu^{2}=\lambda_{2} x^{2}+\frac{\lambda_{3}}{2} v^{2}$.

Since the denominator in Eqs. (39)-(40) is always positive (assuming that the potential is well-defined), the numerators need to be positive as well in order to guarantee a positivedefinite non-vanishing solution for $v$ and $x$.
For the determination of the extrema we evaluate the Hessian matrix:

$\mathcal{H}(v, x) \equiv\left(\begin{array}{ll}\frac{\partial^{2} V}{\partial y^{2}} & \frac{\partial^{2} V}{\partial v \partial x} \\ \frac{\partial^{2} V}{\partial v \partial x} & \frac{\partial^{2} V}{\partial x^{2}}\end{array}\right)=\left(\begin{array}{ll}2 \lambda_{1} v^{2} & \lambda_{3} v x \\ \lambda_{3} v x & 2 \lambda_{2} x^{2}\end{array}\right)$.

From this equation, it is straightforward to verify that the solutions are minima if and only if Eqs. (4) and (5) are satisfied.

\section{Appendix B: RGEs for SM gauge couplings and the top quark Yukawa coupling}

This section basically follows the discussion in Ref. [41]. In the SM, all one-loop RGEs for gauge couplings are of the form

$\frac{d x}{d t}=a x^{2}$.

The exact analytic solution for this equation is given by

$x(t)=\frac{x\left(t=t_{0}\right)}{1-a x\left(t=t_{0}\right)\left(t-t_{0}\right)}$,

where for $t=\log \left(\frac{\lambda^{2}}{\lambda_{\text {ref }}^{2}}\right)$ we have

$t-t_{0}=2 \log \left(\frac{\lambda}{\lambda_{0}}\right)$.

For positive values of $a$, the coupling reaches the Landau pole when the denominator in Eq. (43) goes to 0; for negative values, $x \rightarrow 0$ for $t \rightarrow \infty$.

The Yukawa coupling terms are in turn given by

$\frac{d x}{d t}=a x+b x^{3}$

with the solution

$x(t)=\frac{\sqrt{a C^{\prime}\left(t_{0}\right)} e^{a\left(t-t_{0}\right)}}{\sqrt{1-b e^{2 a\left(t-t_{0}\right)} C^{\prime}\left(t_{0}\right)}}$,

with $C^{\prime}\left(t_{0}\right)=\frac{x_{0}^{2}}{a+b x_{0}^{2}}$, where $x\left(t=t_{0}\right) \equiv x_{0}$ defines the initial value. For the top quark Yukawa coupling we have

$16 \pi^{2} a=-4 g_{s}^{2}-\frac{9}{8} g^{2}-\frac{17}{24} g^{\prime 2}$,

$16 \pi^{2} b=\frac{9}{4}$.

However, taking the explicit scale dependence of the SM gauge couplings into account, the above solution needs to be modified such that $a\left(t-t_{0}\right)$ is replaced by $\int_{t_{0}}^{t} a\left(t^{\prime}\right) d t^{\prime}$. In this work we chose to solve the RGE of the top quark Yukawa coupling numerically. 


\section{References}

1. ATLAS Collaboration, G. Aad et al., Phys. Lett. B 716, 1 (2012). arXiv: 1207.7214

2. CMS Collaboration, S. Chatrchyan et al., Phys. Lett. B 716, 30 (2012). arXiv:1207.7235

3. ATLAS Collaboration, G. Aad et al., Phys. Lett. B 726, 120 (2013). arXiv:1307.1432

4. ATLAS Collaboration, G. Aad et al. (2014). arXiv:1408.7084

5. ATLAS Collaboration, G. Aad et al. (2014). arXiv:1408.5191

6. ATLAS Collaboration, G. Aad et al. (2014). arXiv:1409.6212

7. CMS Collaboration, V. Khachatryan et al., Eur. Phys. J. C 74, 3076 (2014). arXiv: 1407.0558

8. CMS Collaboration, S. Chatrchyan et al., Nat. Phys. 10 (2014). arXiv: 1401.6527

9. CMS Collaboration, S. Chatrchyan et al., Phys. Rev. D 89, 092007 (2014). arXiv: 1312.5353

10. CMS Collaboration, S. Chatrchyan et al., JHEP 1401, 096 (2014). arXiv: 1312.1129

11. P.W. Higgs, Phys. Lett. 12, 132 (1964)

12. P.W. Higgs, Phys. Rev. Lett. 13, 508 (1964)

13. F. Englert, R. Brout, Phys. Rev. Lett. 13, 321 (1964)

14. G. Guralnik, C. Hagen, T. Kibble, Phys. Rev. Lett. 13, 585 (1964)

15. T. Kibble, Phys. Rev. 155, 1554 (1967)

16. ATLAS Collaboration, G. Aad et al., Phys. Rev. D 90, 052004 (2014). arXiv:1406.3827

17. CMS Collaboration (2014). CMS-PAS-HIG-14-009

18. D. Asner et al. (2013). arXiv: 1310.0763

19. R. Schabinger, J.D. Wells, Phys. Rev. D 72, 093007 (2005). arXiv:hep-ph/0509209

20. B. Patt, F. Wilczek (2006). arXiv:hep-ph/0605188

21. V. Barger, P. Langacker, M. McCaskey, M.J. Ramsey-Musolf, G. Shaughnessy, Phys. Rev. D 77, 035005 (2008). arXiv:0706.4311

22. G. Bhattacharyya, G.C. Branco, S. Nandi, Phys. Rev. D 77, 117701 (2008). arXiv:0712.2693

23. S. Dawson, W. Yan, Phys. Rev. D 79, 095002 (2009). arXiv:0904.2005

24. S. Bock et al., Phys. Lett. B 694, 44 (2010). arXiv:1007.2645

25. P.J. Fox, D. Tucker-Smith, N. Weiner, JHEP 1106, 127 (2011). arXiv: 1104.5450

26. C. Englert, T. Plehn, D. Zerwas, P.M. Zerwas, Phys. Lett. B 703, 298 (2011). arXiv:1106.3097

27. C. Englert, J. Jaeckel, E. Re, M. Spannowsky, Phys. Rev. D 85, 035008 (2012). arXiv:1111.1719

28. B. Batell, S. Gori, L.-T. Wang, JHEP 1206, 172 (2012). arXiv: 1112.5180

29. C. Englert, T. Plehn, M. Rauch, D. Zerwas, P.M. Zerwas, Phys. Lett. B 707, 512 (2012). arXiv:1112.3007

30. R.S. Gupta, J.D. Wells, Phys. Lett. B 710, 154 (2012). arXiv: 1110.0824

31. M.J. Dolan, C. Englert, M. Spannowsky, Phys. Rev. D 87, 055002 (2013). arXiv:1210.8166

32. D. Bertolini, M. McCullough, JHEP 1212, 118 (2012). arXiv: 1207.4209

33. B. Batell, D. McKeen, M. Pospelov, JHEP 1210, 104 (2012). arXiv: 1207.6252

34. D. Lopez-Val, T. Plehn, M. Rauch, JHEP 1310, 134 (2013). arXiv: 1308.1979

35. The LHC Higgs Cross Section Working Group, S. Heinemeyer et al. (2013). arXiv:1307.1347

36. R.S. Chivukula, A. Farzinnia, J. Ren, E.H. Simmons, Phys. Rev. D 88, 075020 (2013). arXiv:1307.1064

37. C. Englert, M. McCullough, JHEP 1307, 168 (2013). arXiv: 1303.1526
38. B. Cooper, N. Konstantinidis, L. Lambourne, D. Wardrope, Phys. Rev. D 88, 114005 (2013). arXiv: 1307.0407

39. C. Caillol, B. Clerbaux, J.-M. Frere, S. Mollet, Eur. Phys. J. Plus 129, 93 (2014). arXiv: 1304.0386

40. R. Coimbra, M.O. Sampaio, R. Santos, Eur. Phys. J. C 73, 2428 (2013). arXiv:1301.2599

41. G.M. Pruna, T. Robens, Phys. Rev. D 88, 115012 (2013). arXiv: 1303.1150

42. S. Dawson et al. (2013). arXiv:1310.8361

43. D. Lopez-Val, T. Robens, Phys. Rev. D 90, 114018 (2014). arXiv: 1406.1043

44. C. Englert, M. Spannowsky, Phys. Rev. D 90, 053003 (2014). arXiv: 1405.0285

45. C. Englert, Y. Soreq, M. Spannowsky (2014). arXiv:1410.5440

46. C.-Y. Chen, S. Dawson, I. Lewis (2014). arXiv:1410.5488

47. D. Karabacak, S. Nandi, S.K. Rai, Phys. Lett. B 737, 341 (2014). arXiv: 1405.0476

48. S. Profumo, M.J. Ramsey-Musolf, C.L. Wainwright, P. Winslow (2014). arXiv:1407.5342

49. L. Basso, S. Moretti, G.M. Pruna, Phys. Rev. D 82, 055018 (2010). arXiv: 1004.3039

50. M.J. Strassler, K.M. Zurek, Phys. Lett. B 651, 374 (2007) arXiv:hep-ph/0604261

51. M.J. Strassler, K.M. Zurek, Phys. Lett. B 661, 263 (2008). arXiv:hep-ph/0605193

52. P. Bechtle, O. Brein, S. Heinemeyer, G. Weiglein, K.E. Williams, Comput. Phys. Commun. 181, 138 (2010). arXiv:0811.4169

53. P. Bechtle, O. Brein, S. Heinemeyer, G. Weiglein, K.E. Williams, Comput. Phys. Commun. 182, 2605 (2011). arXiv:1102.1898

54. P. Bechtle et al., PoS CHARGED2012, 024 (2012). arXiv: 1301.2345

55. P. Bechtle et al., Eur. Phys. J. C 74, 2693 (2013). arXiv:1311.0055

56. P. Bechtle, S. Heinemeyer, O. Stål, T. Stefaniak, G. Weiglein, Eur. Phys. J. C 74, 2711 (2014). arXiv:1305.1933

57. P. Bechtle, S. Heinemeyer, O. Stål, T. Stefaniak, G. Weiglein, JHEP 1411, 039 (2014). arXiv:1403.1582

58. M. Bowen, Y. Cui, J.D. Wells, JHEP 0703, 036 (2007). arXiv:hep-ph/0701035

59. G. Altarelli, R. Barbieri, Phys. Lett. B 253, 161 (1991)

60. M.E. Peskin, T. Takeuchi, Phys. Rev. Lett. 65, 964 (1990)

61. M.E. Peskin, T. Takeuchi, Phys. Rev. D 46, 381 (1992)

62. I. Maksymyk, C. Burgess, D. London, Phys. Rev. D 50, 529 (1994). arXiv:hep-ph/9306267

63. B.W. Lee, C. Quigg, H. Thacker, Phys. Rev. D 16, 1519 (1977)

64. M. Luscher, P. Weisz, Phys. Lett. B 212, 472 (1988)

65. L. Basso, A. Belyaev, S. Moretti, G. Pruna, Phys. Rev. D 81, 095018 (2010). arXiv: 1002.1939

66. R.N. Lerner, J. McDonald, Phys. Rev. D 80, 123507 (2009). arXiv:0909.0520

67. G. Degrassi et al., JHEP 1208, 098 (2012). arXiv:1205.6497

68. R. Costa, A.P. Morais, M.O.P. Sampaio, R. Santos (2014). arXiv: 1411.4048

69. ALEPH Collaboration, DELPHI Collaboration, L3 Collaboration, OPAL Collaboration, LEP Electroweak Working Group, J. Alcaraz et al. (2006). arXiv:hep-ex/0612034

70. CDF Collaboration, T. Aaltonen et al., Phys. Rev. Lett. 108, 151803 (2012). arXiv: 1203.0275

71. D0 Collaboration, V.M. Abazov et al., Phys. Rev. D 89, 012005 (2014). arXiv:1310.8628

72. M. Awramik, M. Czakon, A. Freitas, G. Weiglein, Phys. Rev. D 69, 053006 (2004). arXiv:hep-ph/0311148

73. Gfitter Group, M. Baak et al., Eur. Phys. J. C 74, 3046 (2014). arXiv: 1407.3792

74. K. Hagiwara, S. Matsumoto, D. Haidt, C. Kim, Z. Phys. C 64, 559 (1994). arXiv:hep-ph/9409380 
75. S. Goria, G. Passarino, D. Rosco, Nucl. Phys. B 864, 530 (2012). arXiv: 1112.5517

76. K. Hagiwara et al., Phys. Rev. D 73, 055005 (2006). arXiv:hep-ph/0512260

77. C. Uhlemann, N. Kauer, Nucl. Phys. B 814, 195 (2009). arXiv:0807.4112

78. D. Wiesler, DESY-THESIS-2012-048

79. E. Maina (2015). arXiv: 1501.02139

80. H.E. Logan (2014). arXiv: 1412.7577

81. J. Kalinowski, W. Kilian, J. Reuter, T. Robens, K. Rolbiecki, JHEP 0810, 090 (2008). arXiv:0809.3997

82. N. Kauer, G. Passarino, JHEP 1208, 116 (2012). arXiv:1206.4803

83. CMS Collaboration, S. Chatrchyan et al., Phys. Lett. B 710, 26 (2012). arXiv:1202.1488

84. ATLAS Collaboration, G. Aad et al., Phys. Rev. D 86, 032003 (2012). arXiv:1207.0319

85. CMS Collaboration (2012). CMS-PAS-HIG-12-045

86. ATLAS Collaboration (2013). ATLAS-CONF-2013-013, ATLAS-COM-CONF-2013-018

87. CMS Collaboration, S. Chatrchyan et al., Phys. Rev. Lett. 108, 111804 (2012). arXiv:1202.1997

88. CMS Collaboration (2013). CMS-PAS-HIG-13-002

89. ATLAS Collaboration (2013). ATLAS-CONF-2013-030, ATLAS-COM-CONF-2013-028

90. CMS Collaboration (2013). CMS-PAS-HIG-13-003

91. ATLAS Collaboration, G. Aad et al., Phys. Rev. Lett. 113, 171801 (2014). arXiv:1407.6583

92. ALEPH Collaboration, DELPHI Collaboration, L3 Collaboration, OPAL Collaboration, LEP Working Group for Higgs Boson Searches, S. Schael et al., Eur. Phys. J. C 47, 547 (2006). arXiv:hep-ex/0602042

93. OPAL Collaboration, G. Abbiendi et al., Eur. Phys. J. C 27, 311 (2003). arXiv:hep-ex/0206022

94. CMS Collaboration (2013). CMS-PAS-HIG-13-010

95. CMS Collaboration (2013). CMS-PAS-HIG-13-025

96. ATLAS Collaboration (2014). ATLAS-CONF-2014-060, ATLAS-COM-CONF-2014-078

97. ATLAS Collaboration (2014). ATLAS-CONF-2014-061, ATLAS-COM-CONF-2014-080

98. Y. Alexahin et al. (2013). arXiv:1308.2143

99. CMS Collaboration (2014). CMS-PAS-HIG-13-032
100. CMS Collaboration (2014). CMS-PAS-HIG-14-013

101. LHC Higgs Cross Section Working Group, S. Dittmaier et al. (2011). arXiv:1101.0593

102. S. Dittmaier et al. (2012). arXiv: 1201.3084

103. F. Wilczek, Phys. Rev. Lett. 39, 1304 (1977)

104. Particle Data Group, K. Olive et al., Chin. Phys. C 38, 090001 (2014)

105. M. Vysotsky, Phys. Lett. B 97, 159 (1980)

106. P. Nason, Phys. Lett. B 175, 223 (1986)

107. J.F. Gunion, H.E. Haber, G.L. Kane, S. Dawson, Front. Phys. 80, 1 (2000)

108. BaBar Collaboration, B. Aubert et al., Phys. Rev. Lett. 103, 181801 (2009). arXiv:0906.2219

109. BaBar Collaboration, J. Lees et al., Phys. Rev. D 88, 071102 (2013). arXiv: 1210.5669

110. BaBar Collaboration, B. Aubert et al., Phys. Rev. Lett. 103, 081803 (2009). arXiv:0905.4539

111. BaBar collaboration, J. Lees et al., Phys. Rev. D 87, 031102 (2013). arXiv: 1210.0287

112. BaBar Collaboration, J. Lees et al., Phys. Rev. D 88, 031701 (2013). arXiv:1307.5306

113. CLEO Collaboration, W. Love et al., Phys. Rev. Lett. 101, 151802 (2008). arXiv:0807.1427

114. R. Dermisek, J.F. Gunion, Phys. Rev. Lett. 95, 041801 (2005). arXiv:hep-ph/0502105

115. R. Dermisek, J.F. Gunion, B. McElrath, Phys. Rev. D 76, 051105 (2007). arXiv:hep-ph/0612031

116. F. Domingo, U. Ellwanger, E. Fullana, C. Hugonie, M.-A. Sanchis-Lozano, JHEP 0901, 061 (2009). arXiv:0810.4736

117. B. Echenard, Adv. High Energy Phys. 2012, 514014 (2012). arXiv: 1209.1143

118. F. Domingo, JHEP 1104, 016 (2011). arXiv: 1010.4701

119. BaBar Collaboration, Belle Collaboration, A. Bevan et al., Eur. Phys. J. C 74, 3026 (2014). arXiv:1406.6311

120. Belle-II Collaboration, T. Abe et al. (2010). arXiv:1011.0352

121. M. Grazzini. Private communication

122. ATLAS Collaboration (2014). ATLAS-CONF-2014-005, ATLAS-COM-CONF-2014-007 